\title{
Optimal transportation networks and stations
}

\author{
TUOMO VALKONEN \\ Department of Mathematical Information Technology, University of Jyväskylä, \\ FIN-40100 University of Jyväskylä, Finland \\ E-mail: tujumava@jyu.fi
}

[Received 19 February 2009]

\begin{abstract}
We study a model of optimal transportation networks that includes the cost of constructing stations. The model is expressed in terms of the Federer-Fleming language of currents.
\end{abstract}

\section{Introduction}

Various authors consider the Monge-Kantorovich problem of irrigating a signed measure; see, e.g., Ambrosio [1] for an overview. Given $\mu \triangleq \mu^{+}-\mu^{-}$with $\mu^{+}\left(\mathbb{R}^{m}\right)=\mu^{-}\left(\mathbb{R}^{m}\right)<\infty$ to be irrigated, one way to formulate the problem is to find a transport plan $\pi$ that attains

$$
\min \left\{\int c(x, y) \mathrm{d} \pi(x, y) \mid \pi_{1}=\mu^{-}, \pi_{2}=\mu^{+}\right\} .
$$

Here $c$ is a given lower semicontinuous cost function, and $\pi_{i}$ are the marginals of $\pi$. In the typical case $c(x, y)=\|x-y\|^{p}, p \geqslant 1$, and the $p$ th root of the minimum in (1.1) is then called the $p$-Wasserstein distance $\mathcal{W}_{p}\left(\mu^{+}, \mu^{-}\right)$between $\mu^{+}$and $\mu^{-}$.

Brancolini and Buttazzo [6], Buttazzo and Stepanov [8], and Buttazzo et al. [7], among other works, consider a variant of the problem with two different media. The object is then to find a onedimensional network or track $\Sigma$ that attains 1.1 when the cost $c$ of travelling between $x$ and $y$ is defined in such a way that it is cheaper to travel on $\Sigma$ than outside it.

Another variant of the problem is considered by Bernot et al. [5], and Xia [17, 18, 19], among others. Although employing a single medium, this version also attempts to include the cost of building the transportation network by considering a target functional that attempts to group together the paths taken by mass travelling from $\mu^{+}$to $\mu^{-}$, forming a rectifiable network. In the language of Federer-Fleming currents, this can be formulated as finding a current $T$ of optimal weighted area $\mathbb{M}^{\alpha}(T)(\alpha \in[0,1))$ such that $\partial T=\mu$.

Paolini and Stepanov [14] also study a variant of the two-media problem in the language of currents: that of minimising the functional

$$
(W, R) \mapsto A \mathbb{M}^{\alpha}(W)+B \mathbb{M}^{\beta}(R)+H\left(\mathbb{M}^{\delta}(R)\right) \quad \text { with } \quad \partial(W+R)=\mu .
$$

The first term of the functional describes the cost of the portion of paths that are to be travelled by "own means", the second term describes the cost of travelling the network modelled by the real rectifiable current $R$, and the last term describes the cost of constructing this network.

In application to construction of (small scale) urban transportation networks, none of these models is yet entirely realistic. One apparent flaw, which we intend to remedy in this paper, is that they do not take into account the cost of constructing stations or stops, which are nicely modelled 
by $\partial R$ in the language of currents. Indeed, $\partial R$ may have infinite mass, or even be unrectifiable, the corresponding measure being absolutely continuous with respect to the Lebesgue measure. Traffic may enter the network from anywhere. The next examples demonstrate these problems.

EXAMPLE 1.1 Let $x_{1}=0<x_{2}<\cdots<x_{\infty}=1, x_{i} \nearrow 1$. Set $\mu^{-} \triangleq \delta_{x_{1}}+(2 / 3) \sum_{i=1}^{\infty} 2^{-i} \delta_{x_{2 i+1}}$, and $\mu^{+} \triangleq \sum_{i=1}^{\infty} 2^{-i} \delta_{x_{2 i}}+(2 / 3) \delta_{x_{\infty}}$. Then $\mu^{+}(\mathbb{R})=\mu^{-}(\mathbb{R})=1+2 / 3<\infty$, and the optimal current connecting $\mu^{+}$with $\mu^{-}$is $T=\sum_{i=1}^{\infty}\left[\phi_{i} \llbracket x_{2 i-1}, x_{2 i} \rrbracket+\left(\phi_{i}-2^{-i}\right) \llbracket x_{2 i}, x_{2 i+1} \rrbracket\right]$, where $\phi_{i} \triangleq 1-(1 / 3) \sum_{j=1}^{i-1} 2^{-j}$. This is because some mass must be transported from $x_{1}$ to $x_{\infty}$, and $T$ must be supported in the convex hull of the support of $\mu$. In the model (1.2), $T$ splits into $W$ and $R$ by a parameter-dependent threshold on the density of $T$ [14, Theorem 10.4], which alternates here above and below $2 / 3=\lim _{i \rightarrow \infty} \phi_{i}$, with $\phi_{i}-2^{-i}<2 / 3<\phi_{i}$. Therefore, a suitable choice of parameters ensures $R=\sum_{i=1}^{\infty} \phi_{i} \llbracket x_{2 i-1}, x_{2 i} \rrbracket$. But then $\mathbb{M}(\partial R)=2 \sum_{i=1}^{\infty} \phi_{i}=\infty$.

EXAMPLE 1.2 Let $\mu \triangleq \operatorname{id}\left(\mathcal{H}^{1}\llcorner[-1,1] \times\{0\})\right.$ in $\mathbb{R}^{2}$. Then the corresponding optimal current must be $T \triangleq \llbracket(-1,0),(1,0) \rrbracket\left\llcorner\theta\right.$, where $\theta(t)=\int_{-1}^{t}-s \mathrm{~d} s=\left(1-t^{2}\right) / 2$. As above, $R=T\llcorner\{\theta \geqslant \alpha\}$ for some $\alpha \geqslant 0$. If $\alpha<1 / 2$, then for $\tau \triangleq \sqrt{1-2 \alpha}, \partial R(\omega)=\int_{-\tau}^{\tau} \omega^{\prime}(t) \theta(t) \mathrm{d} t=\alpha[\omega(\tau)-\omega(-\tau)]+$ $\int_{-\tau}^{\tau} \omega(s) s \mathrm{~d} s$, which is unrectifiable, not being a countable sum of Dirac masses.

Our task in this paper is thus to add a cost for $\partial R$, and to study the properties that follow for $R$.

The rest of this paper is laid out as follows. First in Section 2, we present some background information on currents and transportation problems. Then, in Section 3, we study notions of connectedness of currents. This section forms the main part of the paper, and allows us to work in a rather abstract and even discrete manner in the following Section 4 There we introduce our cost functional in a general form and study the properties of optimal $R$ that follow under some abstract assumptions. In the final Section 5 we provide a more detailed analysis of the problem when the cost functional has a specific form employing weighted areas $\mathbb{M}^{\alpha}$. In Appendix $\mathrm{A}$ we also consider connectedness in a special case related to functions of bounded variation, and previous notions of indecomposability for sets of finite perimeter.

\section{Preliminaries}

\subsection{Basics on currents}

Following Federer [11] or Morgan [13], a $k$-dimensional current $T \in \mathcal{D}_{k}\left(\mathbb{R}^{m}\right)$ is defined as a linear functional on $k$-dimensional differential forms in $\mathbb{R}^{m}$. When $k>1$, the boundary is the $k-1$ dimensional current defined by $\partial T(\omega) \triangleq T(d \omega)$. The support is the smallest closed set supp $T$ such that $T(\omega)=0$ whenever supp $\omega \cap \operatorname{supp} T=0$. The mass is defined as $\mathbb{M}(T) \triangleq \sup \{T(\omega)$ | $\left.\|\omega\|_{\infty} \leqslant 1\right\} . T$ is representable by integration if $T(\omega)=\tau_{T} \wedge \mu_{T} \triangleq \int\left\langle\omega, \tau_{T}\right\rangle \mathrm{d} \mu_{T}$ for some measure $\mu_{T}$ and a unit vector field $\tau_{T}$. Such a representation exists when $\mathbb{M}(T)<\infty$.

When $\theta$ is a $C^{\infty}$ function, we define $(T\llcorner\theta)(\omega)=T(\theta \wedge \omega)$. When $T$ is representable by integration, this may be extended to other Borel functions. In particular, for the indicator function $\chi_{A}$ of a Borel set $A$, we set $\left(T\llcorner A)(\omega)=T\left(\chi_{A} \wedge \omega\right)=\int_{A}\left\langle\omega, \tau_{T}\right\rangle \mathrm{d} \mu_{T}\right.$.

$T$ is a normal current if the support is compact and $\mathbb{M}(T)+\mathbb{M}(\partial T)<\infty$. If we define the flat norm $\mathbb{F}(T) \triangleq \inf \left\{\mathbb{M}(T-\partial Q)+\mathbb{M}(Q) \mid Q \in \mathcal{D}_{k+1}\left(\mathbb{R}^{m}\right)\right\}$, then the flat chains are the closure of the space of normal currents in this norm.

$T$ is said to be real rectifiable if $\mu_{T}=\theta \mathcal{H}^{k}\llcorner\Sigma$ for a Borel map $\theta$, a rectifiable set $\Sigma$, i.e., a countable union of images of Lipschitz maps, and $\tau_{T}$ a tangent vector field of $\Sigma$. When $\tau_{T}$ is 
implicit, we denote $T=\llbracket \Sigma \rrbracket\llcorner\theta$. If $\Sigma$ is a finite union of $k$-dimensional simplices, and $\theta$ is constant on each simplex, then $T$ is a polyhedral current.

If $\gamma:[0,1] \rightarrow \mathbb{R}^{m}$ is a Lipschitz curve, we define the one-dimensional current $\llbracket \gamma \rrbracket$ by $\llbracket \gamma \rrbracket(\omega) \triangleq \int_{0}^{1}\left\langle\gamma^{\prime}(t), \omega(\gamma(t))\right\rangle \mathrm{d} t$. The result is independent of the parametrisation. We also let $\llbracket x, y \rrbracket \triangleq \llbracket t \mapsto x+t(y-x) \rrbracket$ denote the current corresponding to the straight line segment from $x$ to $y\left(\partial \llbracket x, y \rrbracket=\delta_{y}-\delta_{x}\right)$.

When $S$ is a 0 -dimensional current with finite mass, hence a signed measure, we denote by $S^{+}$ and $S^{-}$the positive and negative parts of $S$ (as a measure).

\subsection{Subcurrents}

Following Paolini and Stepanov [14], we say that $S$ is a subcurrent of $T$, denoted $S \leqslant T$, if

$$
\mathbb{M}(T-S)+\mathbb{M}(S) \leqslant \mathbb{M}(T) .
$$

When $T$ is representable by integration as $T=\tau_{T} \wedge \mu_{T}$, and $S \leqslant T$, there is according to [14, Lemma 3.7] a Borel function $\sigma: \mathbb{R}^{m} \rightarrow[0,1]$ such that $S=T\left\llcorner\sigma=\tau_{T} \wedge \sigma \mu_{T}\right.$.

The current $C$ is a cycle if $\partial C=0 . T$ is said to be acyclic if $C \leqslant T$ being a cycle implies $C=0$.

A circuit of $T$ is a pair of distinct subcurrents $C_{1}, C_{2} \leqslant T$ such that $C_{1}-C_{2}$ is a cycle, i.e., $\partial\left(C_{1}-C_{2}\right)=0 . T$ containing no circuit obviously implies acyclicity.

\subsection{Transport measures}

Let $\gamma_{1}, \gamma_{2}:[0,1] \rightarrow \mathbb{R}^{m}$ be Lipschitz curves. We equip them with the distance

$$
d_{\bar{\Gamma}}\left(\gamma_{1}, \gamma_{2}\right)=\inf \left\{\max _{t \in[0,1]}\left\|\hat{\gamma}_{1}(t)-\hat{\gamma}_{2}(t)\right\| \mid \hat{\gamma}_{i} \text { is a parametrisation of } \gamma_{i}\right\},
$$

where " $\hat{\gamma}$ is a parametrisation of $\gamma$ " means that $\hat{\gamma}$ is a Lipschitz curve satisfying $\hat{\gamma}=\gamma \circ \psi$ for some continuous surjective non-decreasing map $\psi:[0,1] \rightarrow[0,1]$. We often identify curves with the corresponding parametrisation equivalence class, and denote the space of the latter by $\bar{\Gamma}$. Then $d_{\bar{\Gamma}}$ forms a metric on $\bar{\Gamma}$.

We now recall from [14, Theorem 6.3] that an acyclic 1-dimensional normal current $T$ can be represented by a transport measure, in the form

$$
T(\omega)=\llbracket \eta \rrbracket(\omega) \triangleq \int \llbracket \gamma \rrbracket(\omega) \mathrm{d} \eta(\gamma) .
$$

Here $\eta$ is a Borel measure over $\bar{\Gamma}$ such that $\eta_{0}=(\partial T)^{-}, \eta_{1}=(\partial T)^{+}$, and $\eta_{i}(D) \triangleq \eta\{\gamma$ | $\gamma(i) \in D\}=\left(p_{i}\right)_{\# \eta} \eta(D), p_{i}(\gamma) \triangleq \gamma(i)$ for Borel sets $D$. In fact, we may take the $\gamma$ to be a.e. arcs, i.e., non-self-crossing.

We then have $\mu_{T}(D)=\int \mathbb{M}\left(\llbracket \gamma \rrbracket\llcorner D) \mathrm{d} \eta(\gamma)\right.$ and $T\left\llcorner D(\omega)=\int \llbracket \gamma \rrbracket\llcorner D(\omega) \mathrm{d} \eta(\gamma)\right.$ for Borel sets $D$. Also every restriction of the transport integral on a Borel set $\Gamma$ of (equivalence classes of) curves, forms a subcurrent $\omega \mapsto \int_{\Gamma} \llbracket \gamma \rrbracket(\omega) \mathrm{d} \eta(\gamma)$ of $R$.

We recall that the transiting mass function is defined as $\alpha_{\eta}(x) \triangleq \eta(\{\gamma \mid x \in \operatorname{img} \gamma\})$, and that $\theta=\alpha_{\eta} \mathcal{H}^{1}$-a.e. whenever $T=\llbracket \eta \rrbracket=\llbracket \Sigma \rrbracket\llcorner\theta$ is normal real rectifiable and acyclic [14, Lemmas 7.1 $\& 7.2]$.

We will occasionally be stopping curves upon entrance or leaving a closed set, along the theme of the next lemma. 
LEMMA 2.1 Let $D$ be a closed set, and suppose $\bar{\Gamma}$ is equipped with a $\sigma$-finite measure. Then there exists an almost everywhere defined Borel measurable extension to $\bar{\Gamma}$ of the map $v: \gamma \mapsto$ $\gamma\llcorner[0, \inf \{t \geqslant 0 \mid \gamma(t) \in D\}]$, when for $a \leqslant b$ and a Lipschitz curve $\gamma$, we define

$$
\gamma\llcorner[a, b]: t \in[0,1] \mapsto \gamma(\max \{a, \min \{t, b\}\}) .
$$

Proof. We first consider the measurability of $v$ on individual curves (instead of equivalence classes), with metric $d\left(\gamma_{1}, \gamma_{2}\right) \triangleq \max _{t \in[0,1]}\left\|\gamma_{1}(t)-\gamma_{2}(t)\right\|$. We define the entrance time $\tau(\gamma)=$ $\inf \{t \geqslant 0 \mid \gamma(t) \in D\}$, the helper function $f(\gamma, t)(s) \triangleq \gamma(\min \{s, t\})$, and the stopped path $v(\gamma) \triangleq f(\gamma, \tau(\gamma))$. The entrance time $\tau$ is upper-semicontinuous and hence measurable: If $\max _{t \in[0,1]}\left\|\gamma_{k}(t)-\gamma(t)\right\| \rightarrow 0$, then (for a subsequence) $D \ni \gamma_{k}\left(\tau\left(\gamma_{k}\right)\right) \rightarrow \gamma\left(\lim _{k} \tau\left(\gamma_{k}\right)\right)$. Since $D$ is closed, $\gamma\left(\lim _{k} \tau\left(\gamma_{k}\right)\right) \in D$, so $\lim _{k} \tau\left(\gamma_{k}\right) \geqslant \tau(\gamma)$. The transformation $v$ is therefore measurable as a function on individual Lipschitz curves. Since $f$ is a Carathéodory function, being continuous in each variable separately with the other fixed, and the space of Lipschitz curves is a subset of the complete separable space of continuous functions, the transformation $v$ is thus measurable as a function on individual Lipschitz curves (see, e.g., [4, Lemma 8.2.3]).

Finally, we extend $v$ to equivalence classes as $g \circ v \circ h$, where $g: \gamma \mapsto \bar{\gamma}$ maps $\gamma$ to the corresponding equivalence class $\bar{\gamma}$, and $h$ is a selection of $g^{-1}$. The function $g$ is continuous, hence measurable. We want $h$ to be measurable as well. Towards that end, note that the graph of $g$ is measurable by the continuity of $g$. Also, the set of Lipschitz paths is a Borel measurable subset of the complete separable space of continuous functions (every subset of curves with Lipschitz factor bounded by a given constant being closed). Hence, by the Neumann-Aumann measurable selection theorem (see, e.g., [12, 9]), there is a measurable function $h$ such that $h(\bar{\gamma}) \in g^{-1}(\bar{\gamma})$ a.e. Therefore $g \circ v \circ h$ is measurable.

\section{Notions of connectedness of currents}

\subsection{Intersections and unions of currents}

DEFINITION 3.1 Given two finite-mass currents $S$ and $T$, we define the intersection $S \wedge T$ as the greatest current $R$ (in the subcurrent order) such that $R \leqslant S, T$. Likewise, when $S, T \leqslant S+T$, the union $S \vee T$ is defined to be the smallest current $R$ such that $S, T \leqslant R$.

LEMMA 3.1 $S \wedge T$ and $S \vee T$ are well-defined. Furthermore, $S \vee T=S+T-S \wedge T$ when defined.

Proof. Since the currents are assumed to have finite mass, $S=\tau_{S} \wedge \mu_{S}$ and $T=\tau_{T} \wedge \mu_{T}$ can be represented by integration. Consequently, any current $R$ such that $R \leqslant S, T$ has $R=\tau_{S} \wedge \sigma_{S} \mu_{S}=$ $\tau_{T} \wedge \sigma_{T} \mu_{T}$ for some Borel maps $\sigma_{S}, \sigma_{T}: \mathbb{R}^{m} \rightarrow[0,1]$. This says that $R \leqslant \hat{R} \triangleq \tau_{S} \wedge\left[\left(\mu_{S} \wedge \mu_{T}\right)\llcorner D]\right.$, where $D \triangleq\left\{x \in \mathbb{R}^{m} \mid \tau_{S}(x)=\tau_{T}(x)\right\}$. We must then have $S \wedge T=\hat{R}$, which means that it is well-defined.

As for $S \vee T$, suppose $S, T \leqslant R \leqslant S+T$. Then

$$
S+T-R \leqslant S, T
$$

as follows from applying the definition of subcurrents: Combining $\mathbb{M}(R)+\mathbb{M}(R-(S+T)) \leqslant$ $\mathbb{M}(S+T)$ and $\mathbb{M}(R-T)+\mathbb{M}(T) \leqslant \mathbb{M}(R)$ gives $\mathbb{M}(R-T)+\mathbb{M}(R-(S+T)) \leqslant \mathbb{M}(S+T)-\mathbb{M}(T) \leqslant$ $\mathbb{M}(S)$, which is just $S+T-R \leqslant S$. The proof for $T$ is analogous. The minimal current $R$ for which (3.1) can hold is given by $S+T-R=S \wedge T$. 
REMARK 3.1 If $S_{i}=\tau_{i} \wedge \sigma_{i} \mu_{T}, \sigma_{i} \geqslant 0, i=1,2$, then $S_{1} \wedge S_{2}=\tau_{T} \wedge \min \left\{\sigma_{1}, \sigma_{2}\right\} \mu_{T}$, and $S_{1} \vee S_{2}=\tau_{T} \wedge \max \left\{\sigma_{1}, \sigma_{2}\right\} \mu_{T}$.

If $S_{3}$ has a similar expression, one easily observes that also $\left(S_{1} \vee S_{2}\right) \wedge S_{3}=\left(S_{1} \wedge S_{3}\right) \vee\left(S_{2} \wedge S_{3}\right)$ and $\left(S_{1} \wedge S_{2}\right) \wedge S_{3}=\left(S_{1} \wedge S_{3}\right) \wedge\left(S_{2} \wedge S_{3}\right)$. In particular, $\left(S_{1} \vee S_{2}\right)\left\llcorner V=\left(S_{1}\llcorner V) \vee\left(S_{2}\llcorner V)\right.\right.\right.$ and $\left(S_{1} \wedge S_{2}\right)\left\llcorner V=\left(S_{2}\llcorner V) \wedge\left(S_{2}\llcorner V)\right.\right.\right.$ for a Borel set $V$.

Lemma 3.2 Suppose $S_{1}, S_{2} \leqslant T$. Then $T=S_{1} \vee S_{2}+\left(T-S_{1}\right) \wedge\left(T-S_{2}\right)$.

Proof. If $X \leqslant T$, then $S_{1}, S_{2} \leqslant X$ if and only if $T-X \leqslant T-S_{1}, T-S_{2}$ by the definition (2.1) of a subcurrent. Thus, taking $X=S_{1} \vee S_{2}$, we find $T-S_{1} \vee S_{2} \leqslant\left(T-S_{1}\right) \wedge\left(T-S_{2}\right)$. Likewise, taking $X^{\prime} \triangleq T-\left(T-S_{1}\right) \wedge\left(T-S_{2}\right)$, we find $S_{1} \vee S_{2} \leqslant T-\left(T-S_{1}\right) \wedge\left(T-S_{2}\right)$. We thus have $T-X \leqslant T-X^{\prime}$ and $X \leqslant X^{\prime}$. As also $X, X^{\prime} \leqslant T$, we find $\mathbb{M}\left(X-X^{\prime}\right)=0$ or $X=X^{\prime}$ by application of the definition 2.1.

LEMMA 3.3 Suppose $S_{1}, S_{2}$ and $T$ are finite mass currents, and that $S_{1} \vee S_{2}$ is defined. Then $T \wedge\left(S_{1}+S_{2}\right) \leqslant T \wedge S_{1}+T \wedge S_{2}$. If $\left(T \wedge S_{1}\right) \wedge\left(T \wedge S_{2}\right)=0$, then equality holds. In particular, if $S_{1} \wedge T=0$, then $T \wedge\left(S_{1}+S_{2}\right)=T \wedge S_{2}$.

Proof. Since $S \triangleq S_{1} \vee S_{2}$ is defined, we may express $S_{i}=S\left\llcorner\sigma_{i}\right.$, where $\sigma_{i}: \mathbb{R}^{m} \rightarrow[0,1]$ are Borel maps. We may further assume the similar expression $T=S\left\llcorner\sigma_{T}\right.$, possibly after redefining $T$ as $T \wedge\left(S_{1}+S_{2}\right)$, the remainder not playing a role since $T \wedge S_{i}=T \wedge S_{i} \wedge\left(S_{1}+S_{2}\right)$. Therefore $T \wedge\left(S_{1}+S_{2}\right)=S\left\llcorner\min \left\{\sigma_{T}, \sigma_{1}+\sigma_{2}\right\} \leqslant S\left\llcorner\left(\min \left\{\sigma_{T}, \sigma_{1}\right\}+\min \left\{\sigma_{T}, \sigma_{2}\right\}\right)=T \wedge S_{1}+T \wedge S_{2}\right.\right.$.

Now, if $T \wedge S_{1}=0$, clearly $T \wedge\left(S_{1}+S_{2}\right) \leqslant T \wedge S_{2}$ from the first part. For the other direction, recall that $S_{1} \vee S_{2}$ is defined when $S_{1}, S_{2} \leqslant S_{1}+S_{2}$. Therefore, if $X \leqslant S_{2}, T$, then $X \leqslant S_{1}+S_{2}, T$. In particular, with $X \triangleq T \wedge S_{2}$, we find $T \wedge S_{2} \leqslant T \wedge\left(S_{1}+S_{2}\right)$.

Finally, rewriting $T \wedge\left(S_{1}+S_{2}\right)=T \wedge\left(S_{1} \vee S_{2}+S_{1} \wedge S_{2}\right)$ and employing the assumption $0=\left(T \wedge S_{1}\right) \wedge\left(T \wedge S_{2}\right)=T \wedge\left(S_{1} \wedge S_{2}\right)$ in the result of the previous paragraph, we find $T \wedge\left(S_{1}+S_{2}\right)=T \wedge\left(S_{1} \vee S_{2}\right)=\left(T \wedge S_{1}\right) \vee\left(T \wedge S_{2}\right)=\left(T \wedge S_{1}\right)+\left(T \wedge S_{2}\right)-\left(T \wedge S_{1}\right) \wedge\left(T \wedge S_{2}\right)=$ $\left(T \wedge S_{1}\right)+\left(T \wedge S_{2}\right)$.

\subsection{Connectedness by splitting}

DEFINITION 3.2 Suppose $T$ is a current with finite mass, and $V$ is a Borel set, assumed to be $\mathbb{R}^{m}$ if left unspecified.

(i) The subcurrents $A, B \leqslant T$ split $T$ if $A+B=T$ and $A \wedge B=0$.

(ii) The currents $A, B$ primitive-decompose a normal current $S$ in $V$ if they split $S$, are normal, and $\partial A\left\llcorner V, \partial B\left\llcorner V\right.\right.$ split $\partial S\left\llcorner V{ }^{1}\right.$

(iii) Two currents $A, B$ are said to decompose $T$ into a pair of disjoint components in $V$ if $A$ and $B$ split $T\llcorner V$, and every normal subcurrent $S \leqslant T\llcorner V$ is primitive-decomposed in $V$ by $A \wedge S$ and $B \wedge S$.

(iv) The current $T$ is said to be connected in $V$ if $A, B$ decomposing $T$ into a pair of disjoint components in $V$ implies $A=T\llcorner V$ or $B=T\llcorner V$.

(v) A disjoint component $S$ of $T$ in $V$, which is connected in $V$, is called a connected component (of $T$ in $V$ ).

1 The precedence in our notation is $\partial A\llcorner V=(\partial A)\llcorner V$. 
REMARK 3.2 From the definitions, we observe:

(i) $B=T\llcorner V-A$. It is often more convenient to explicitly mention $B$, however.

(ii) If $A, B$ split $T$, then since $(A \wedge S) \wedge(B \wedge S)=S \wedge(A \wedge B)=0$, according to Lemma 3.3, $A \wedge S, B \wedge S$ split $S \leqslant T$. Consequently, one immediately observes that if $A, B$ decompose $T$ into disjoint components in $V$, then $A \wedge S, B \wedge S$ decompose any $S \leqslant T$ into disjoint components in $V$.

(iii) If $T$ is not connected, it consists of at least two disjoint components, given by the $A, B$ violating the definition.

(iv) If $T$ has no non-trivial normal subcurrent, and has non-singleton support, then it is not connected.

REMARK 3.3 The notion of $T$ being connected is less strict than that of an indecomposable current as defined by Federer [11] for integral currents. It amounts to the non-existence of a non-trivial integral current $A \leqslant T$ with $\partial A \leqslant \partial T$. The current of the next example is decomposable.

With regard to the notion of indecomposability of sets of finite perimeter considered in, e.g., Ambrosio et al. [3, 2] and references therein, closer equivalence can be shown, as in this case the subcurrent splitting requirements become superfluous. We relegate this study to Appendix A.

EXAMPLE 3.1 Consider $T \triangleq A+B \triangleq \llbracket-1,0 \rrbracket+\llbracket 1,0 \rrbracket$. Then the individual segments satisfy all the conditions for disjointness, except $\partial A \wedge \partial B=0$. Thus our notion of connectedness allows "traversing $T$ in reverse"; it is akin to connectedness of support sets.

EXAMPLE 3.2 Let $T \triangleq \llbracket(-1,-1),(1,1) \rrbracket+\llbracket(1,-1),(-1,1) \rrbracket$. Then itself $T$ is primitivedecomposed by $A \triangleq \llbracket(-1,-1), 0 \rrbracket+\llbracket 0,(-1,1) \rrbracket$ and $B \triangleq T-A$, but the subcurrent $S \triangleq$ $\llbracket(-1,-1),(1,1) \rrbracket$ is not primitive-decomposed by $A \wedge S=\llbracket(-1,-1), 0 \rrbracket$ and $B \wedge S=\llbracket 0,(1,1) \rrbracket$.

We need to show that the definition of a disjoint component is sound. This is done by the following lemmas.

LEMMA 3.4 Suppose $A$ is a disjoint component of a finite mass current $T$ in $V$, and $C$ a disjoint (resp. connected) component of $A$ in $U \subset V$. Then $C$ is a disjoint (resp. connected) component of $T$ in $U$.

Proof. Let $B=T\llcorner V-A, D=A\llcorner U-C$, and $E=T\llcorner U-C=B\llcorner U+D$. Clearly $C$ and $E$ split $T\llcorner U$, since $C \leqslant A\llcorner U \leqslant T\llcorner U$. Also note that $D \leqslant A\llcorner U$, while $B \wedge D=0$.

Suppose then that $S \leqslant T\llcorner U$ is normal. Then $C \wedge S=C \wedge(A \wedge S)$ is also normal, since $A$ primitive-decomposes $S$ in $U \subset V$, whence $A \wedge S \leqslant A\llcorner U$ is normal and therefore primitivedecomposed by $C$. Likewise $E \wedge S=B \wedge S+D \wedge S$ is normal, after noting that $(B\llcorner U) \wedge S=$ $B \wedge(S\llcorner U)=B \wedge S$.

By the same primitive-decomposition properties, we also have $\partial(C \wedge S)\llcorner U=\partial(C \wedge$ $(A \wedge S))\llcorner U \leqslant \partial(A \wedge S)\llcorner U \leqslant \partial S\llcorner U$. Likewise $\partial(D \wedge S)\llcorner U \leqslant \partial(A \wedge S)\llcorner U \leqslant \partial S\llcorner U$, and $\partial((B\llcorner U) \wedge S)\llcorner U=\partial(B \wedge S)\llcorner U \leqslant \partial S\llcorner U$. Therefore, we can also calculate $\partial(B \wedge S) \wedge$ $\partial(D \wedge S)\llcorner U \leqslant \partial(B \wedge S) \wedge \partial(A \wedge S)\llcorner U=0$. Consequently, applying Lemma 3.3, also $\partial(E \wedge S)\llcorner U=\partial(B \wedge S)\llcorner U+\partial(D \wedge S)\llcorner U \leqslant \partial S\llcorner U$.

Finally, Lemma 3.3 also allows us to deduce $\partial(C \wedge S) \wedge \partial(E \wedge S)\llcorner U=\partial(C \wedge S) \wedge \partial(B \wedge S)\llcorner U+$ $\partial(C \wedge S) \wedge \partial(D \wedge S)\llcorner U=0$. The last equality follows because $C$ and $D$ decompose $A$ into disjoint components in $U$, because $\partial(C \wedge S)\llcorner U \leqslant \partial(A \wedge S)\llcorner U$, as shown above, and, finally, because $A$ and $B$ decompose $T$ into disjoint components in $V \supset U$. 
Thus we have shown that $C$ remains a disjoint component of $T$ in $U$. Since the definition of connectedness of $C$ in $U$ does not depend on $T$, it remains a connected component of $T$ in $U$.

REMARK 3.4 In particular, taking $V=U$, we find that a disjoint component of a disjoint component of $T$, is a disjoint component of $T$ (all in $V$ ).

LEMMA 3.5 Suppose $A_{i}, B_{i}$ decompose the finite mass current $T$ into disjoint components in the Borel set $V_{i}$ for $i=1,2$. Then $A_{1} \wedge A_{2}$ is a (possibly zero) disjoint component of $T$ in $V \triangleq V_{1} \cap V_{2}$. The same applies to $\left(A_{1} \vee A_{2}\right)\llcorner V$.

Proof. As noted in Remark 3.2, $A_{1} \wedge A_{2}$ is a disjoint component of $A_{2}$ in $V_{1}$, hence also $V \subset V_{1}$. By Lemma 3.4. $A_{1} \wedge A_{2}$ is then a disjoint component of $T$ in $V$.

The claim on the union follows from the claim on the intersection. Applying Lemma 3.2 in the second equality, we find

$$
\begin{aligned}
\left(A_{1} \vee A_{2}\right)\llcorner V & =\left(A _ { 1 } \llcorner V ) \vee \left(A_{2}\llcorner V)=T\left\llcorner V-\left(T \left\llcornerV-A_{1}\llcorner V) \wedge\left(T \left\llcornerV-A_{2}\llcorner V)\right.\right.\right.\right.\right.\right.\right. \\
& =T\left\llcorner V-\left(T \llcorner V _ { 1 } - A _ { 1 } ) \wedge \left(T \llcorner V _ { 2 } - A _ { 2 } ) \left\llcornerV=T\left\llcorner V-B_{1} \wedge B_{2} .\right.\right.\right.\right.\right.
\end{aligned}
$$

But $B_{1} \wedge B_{2}$ is a disjoint component of $T$ in $V$ by the first part.

LEMMA 3.6 Suppose $T_{1}$ and $T_{2}$ are finite mass currents connected in $V$, and that $T_{1} \vee T_{2}$ is defined. If there are $S_{i} \leqslant T_{i}\left\llcorner V, i=1,2\right.$, such that either $S_{1} \wedge S_{2} \neq 0$, or $S_{i}$ are normal and $\left(\partial S_{1} \wedge \partial S_{2}\right)\llcorner V$ $\neq 0$, then $T_{1} \vee T_{2}$ is connected in $V$.

Proof. Let $T \triangleq T_{1} \vee T_{2}$. Suppose $A, B \leqslant T\llcorner V$ decompose $T$ into disjoint components in $V$. Then (for every $S_{i} \leqslant T_{i} \leqslant T$ ) we find $A \wedge S_{i}=A_{i} \wedge S_{i} \triangleq\left(A \wedge T_{i}\right) \wedge S_{i}$ and $B \wedge S_{i}=B_{i} \wedge S_{i} \triangleq\left(B \wedge T_{i}\right) \wedge S_{i}$. Consequently $A_{i}, B_{i} \leqslant T_{i}\left\llcorner V=T_{i} \wedge\left(T\llcorner V)\right.\right.$ are disjoint components of $T_{i}$ in $V$. But, since $T_{i}$ is connected in $V$, either $A_{i}=T_{i}\left\llcorner V\right.$ or $B_{i}=T_{i}\left\llcorner V\right.$ for both $i=1,2$. We may assume $A_{1}=T_{1}\llcorner V$.

Suppose $B_{2}=T_{2}\left\llcorner V\right.$. Then $A_{2}=A \wedge T_{2}=0$. We have $T_{1}\left\llcorner V=A_{1}=A \wedge T_{1}=A \wedge\left(T-T_{2}-\right.\right.$ $T_{1} \wedge T_{2}$ ), whence Lemma 3.3 allows us to deduce $T_{1}\llcorner V=A \wedge T=A$. Analogously we obtain $T_{2}\llcorner V=B$.

But now $S_{1}=S_{1} \wedge\left(T_{1}\llcorner V)=S_{1} \wedge A\right.$ and $S_{2}=S_{2} \wedge\left(T_{2}\llcorner V)=S_{2} \wedge B\right.$. Therefore $S_{1} \wedge S_{2}=$ $\left(S_{1} \wedge S_{2}\right) \wedge(A \wedge B)=0$, providing a contradiction if $S_{1} \wedge S_{2} \neq 0$. Otherwise, $S \triangleq S_{1}+S_{2}=S_{1} \vee S_{2} \leqslant$ $T\left\llcorner V\right.$ is normal, and $S \wedge A=S_{1}$ and $S \wedge B=S_{2}$. Therefore, since $A$ and $B$ primitive-decompose $S$, $\left(\partial S_{1} \wedge \partial S_{2}\right)\llcorner V=0$, contrary to assumption.

We may consequently assume that also $A_{2}=T_{2}\left\llcorner V\right.$, whence $B_{i}=B \wedge T_{i}=0$ for $i=1,2$. Therefore $B=B \wedge T\left\llcorner V=B \wedge\left(T_{1}+T_{2}-T_{1} \wedge T_{2}\right)\llcorner V=0\right.$, by employing Lemma 3.3 again. Consequently, we must have $A=T\left\llcorner V\right.$, and accordingly $T_{1} \vee T_{2}$ is connected.

LEMmA 3.7 Suppose $T$ has finite mass and $U \subset V$ are Borel sets with $\mu_{T}(V \backslash U)=0$. If $A$ is a disjoint component of $T$ in $V$, then $A$ is a disjoint component of $T$ in $U$. Consequently, if $T$ is connected in $U$, then it is connected in $V$.

Proof. $T\llcorner U$ and 0 trivially decompose $T$ into disjoint components in $U$. Therefore, by Lemma 3.5 , $A \wedge\left(T\llcorner U)=A\left\llcorner U\right.\right.$ is a disjoint component of $T$ in $U=V \cap U$. Now, if $\mu_{T}(V \backslash U)=0$, then $A\llcorner U=A\llcorner V=A$. Therefore $A$ is a disjoint component of $T$ in $U$. The claim on connectedness follows from the definition and the first claim. (Note, however, that $A$ being connected in $V$ does not imply the same in $U$.) 
LEMmA 3.8 Suppose $U \subset V$ are Borel sets such that $\mu_{T}\left(V^{c}\right)=0$. If $A$ is a disjoint component of $T$ in $U$, and $\partial(A \wedge S)\left\llcorner V^{c}=0\right.$ for all normal $S \leqslant T\llcorner U$, then $A$ is a disjoint component of $T$ in $U \cup V^{c}$.

Proof. Clearly $A$ and $T\left\llcorner U-A\right.$ split $T\left\llcorner\left(U \cup V^{c}\right)=T\left\llcorner U\right.\right.$, since $\mu_{T}\left(V^{c}\right)=0$. Also, $\partial(A \wedge S)\llcorner$ $\left(U \cup V^{c}\right)=\partial(A \wedge S)\left\llcorner U+\partial(A \wedge S)\left\llcorner V^{c}=\partial(A \wedge S)\left\llcorner U\right.\right.\right.$, since $U \cap V^{c}=U \backslash V=\emptyset$. Thus the boundary-splitting requirements continue to hold.

\subsection{Connections and edges}

In this subsection, we consider one-dimensional normal currents $T$ with real rectifiable boundary. Thus $\partial T=\sum_{i=1}^{\infty} \alpha_{i} \delta_{x_{i}}, \alpha_{i} \neq 0$. We call the points $x_{i}$ the boundary points of $T$, and use the notation $\operatorname{img} \psi \triangleq\left\{x \mid \alpha \delta_{x} \leqslant \psi, \alpha \neq 0\right\}$ for real rectifiable 0 -dimensional currents $\psi$ of finite mass, such as $\partial T$. This can differ from the support, which is a closed set. We also abuse notation slightly and set $\psi(x) \triangleq \alpha$ whenever $\psi\left\llcorner\{x\}=\alpha \delta_{x}\right.$.

Definition 3.3 Suppose $V$ is an open set, and $T$ a normal current. We then make the following definitions:

(i) A normal acyclic subcurrent $E \leqslant T$ is an edge of $T$ in $V$ if supp $E \subset V$, and $\partial E=\alpha\left(\delta_{x}-\delta_{y}\right)$ for some $x, y \in \mathbb{R}^{m}$ and $\alpha \neq 0$.

(ii) A finite sequence of edges $E_{1}, \ldots, E_{n} \leqslant T$ in $V$ and directions $\tau_{i} \in\{-1,+1\}$ with $\partial E_{i}=$ $\tau_{i} \alpha\left(\delta_{x_{i+1}}-\delta_{x_{i}}\right)$ forms a connection between $x_{1}$ and $x_{n+1}$ in $V$. The number $\alpha>0$ is called the strength of the connection.

(iii) If $\partial T$ is real rectifiable, $T$ is edge-connected in $V$ if there exists a connection in $V$ between every $x, y \in \operatorname{img}(\partial T\llcorner V)$.

REMARK 3.5 The existence of a connection between two points is an equivalence relation, thus in particular transitive.

EXAMPLE 3.3 Consider again the polyhedral 1-dimensional chain $T=\llbracket-1,0 \rrbracket+\llbracket 1,0 \rrbracket$ in $\mathbb{R}$. There exists no single edge between -1 and 1 , but $E_{1} \triangleq \llbracket-1,0 \rrbracket$ and $E_{2} \triangleq \llbracket 1,0 \rrbracket$ connect these points.

LEMmA 3.9 Suppose $T$ is a normal current and $V$ an open set. Then every edge $E$ of $T$ in $V$ is connected in $V$.

Proof. If $A \leqslant T$ is normal, the compact support and finite $\mathbb{M}(\partial A)$ imply $\mu_{\partial A}\left(\mathbb{R}^{m}\right)=0$. Furthermore, if $A \leqslant E$, then since supp $A \subset \operatorname{supp} E \subset V$, we have $A=A\llcorner V$ and $\partial A=\partial A\llcorner V$. Therefore we may assume $V=\mathbb{R}^{m}$.

Now, if $A \leqslant E$ and $E-A$ primitive-decompose $E$, then $\partial A \leqslant \partial E=\alpha\left(\delta_{y}-\delta_{x}\right)$. Thus $\partial A=\beta\left(\delta_{y}-\delta_{x}\right)$ for some $\beta \in[0, \alpha]$. But then from $\partial A \wedge \partial(E-A)=0$ we have $\beta=0$ or $\beta=1$. By the acyclicity of $E, \partial A=0$ implies $A=0$, while $\partial A=\partial E$ implies $A=E$. Thus $E$ is connected.

LEMMA 3.10 Suppose $E$ is an edge with no circuits. Then $E=\alpha \llbracket \gamma \rrbracket$ for a Lipschitz path $\gamma$ and $\alpha>0$.

Proof. Since $E$ is normal, we may express $E=\llbracket \eta \rrbracket$ for a transport measure $\eta$. We claim that $\eta$ must be concentrated on a single path $\gamma$. For, if there were disjoint measurable sets $\Gamma$ and $\Gamma^{\prime}$ with 
$\eta\left\llcorner\Gamma, \eta\left\llcorner\Gamma^{\prime} \neq 0\right.\right.$, then these restrictions would form a circuit in violation of that assumption. It remains to show that such sets can be found if $\eta$ is not concentrated on a single path. Let $\Omega$ be a compact convex set such that supp $E \subset \Omega$, and $\Gamma_{\ell} \triangleq\left\{\gamma \in \bar{\Gamma} \mid \mathcal{H}^{1}(\operatorname{img} \gamma) \leqslant \ell\right.$, supp $\left.\gamma \subset \Omega\right\}$. We may assume $\eta$ is concentrated on $\Gamma_{\ell}$ for some $\ell>0$, for otherwise $\Gamma_{\ell}$ and $\Gamma_{\ell}^{c}$ are the sets we are after. But $\Gamma^{1} \triangleq \Gamma_{\ell}$ is compact [7]. Therefore, we can find a finite cover of open balls, $\left\{U\left(\gamma_{i}, 1\right) \mid\right.$ $i=1, \ldots, n\}$. If there are two balls with indices $i$ and $j$ such that $\eta\left(U\left(\gamma_{i}, 1\right) \backslash U\left(\gamma_{j}, 1\right)\right)>0$ and $\eta\left(U\left(\gamma_{j}, 1\right) \backslash U\left(\gamma_{i}, 1\right)\right)>0$, we have found our sets $\Gamma$ and $\Gamma^{\prime}$. Otherwise $\eta$ is concentrated on $\Gamma^{2} \triangleq B\left(\gamma_{i}, 1\right) \cap \Gamma^{1}$ for some $i$, and we continue by covering $\Gamma^{2}$ with balls of diameter $1 / 2$. By recursively repeating the process this way, eventually $\bigcap_{i=1}^{\infty} \Gamma^{i}=\{\gamma\}$, since $\left\{\Gamma^{i}\right\}$ is a nested sequence of closed sets of diameters tending to zero. Thus $\eta$ is concentrated on $\{\gamma\}$.

Lemma 3.11 Suppose $T$ contains no circuit. Then any connection $E_{1}, \ldots, E_{n}$ in $T$ can be assumed to consist of Lipschitz paths $E_{i}=\alpha \llbracket \gamma_{i} \rrbracket$ with $E_{i} \wedge E_{j}=0$ for $i \neq j$.

Proof. By Lemma $3.10 E_{i}=\alpha \llbracket \gamma_{i} \rrbracket$, while by definition $\partial E_{i}=\tau_{i} \alpha\left(\delta_{x_{i+1}}-\delta_{x_{i}}\right)$. If $\gamma_{i}\left(t_{i}\right)=\gamma_{j}\left(t_{j}\right)$ for $t_{i}, t_{j} \in(0,1)(i \leqslant j)$, we may remove the edges $E_{i+1}, \ldots, E_{j-1}$ from the connection, and replace $E_{i}$ (resp. $E_{j}$ ) by the segment before or after $t_{i}$ depending on the direction $\tau_{i}$.

\subsection{Equivalence}

To show partial equivalence between the notion of connectedness in Definitions 3.2 and 3.3 , we require the following concept of a bundle, and some related results. As can be seen from the proof of Lemma 3.12 below, the notion bears some resemblance to the trunk trees of Bernot et al. [5], but we do not require the single-path property.

DEFINITION 3.4 An acyclic normal current $S$ is called a bundle if there is a point $x \in \mathbb{R}^{m}$ such that $S$ can be primitive-decomposed in $\{x\}^{c}$ into $A$ and $B$ with $\partial A\left\llcorner\{x\}^{c}=-(\partial S)^{-}\left\llcorner\{x\}^{c}\right.\right.$ and $\partial B\left\llcorner\{x\}^{c}=(\partial S)^{+}\left\llcorner\{x\}^{c}\right.\right.$.

A bundle $S \leqslant T\llcorner V$ with $\partial S\llcorner V \leqslant \partial T\llcorner V$ is called a $V$-subbundle.

LEMma 3.12 Suppose $S$ is a one-dimensional bundle. Then there exists a transport measure $\llbracket \eta \rrbracket=S$, and connections in $S$ between all $z \in \mathbb{R}^{m}$ with $\alpha_{\eta}(z)>0$.

Proof. Let $x, A=\llbracket \eta_{A} \rrbracket$, and $B=\llbracket \eta_{B} \rrbracket$ be as in Definition 3.4. For $X=A, B$, we let $m_{X} \triangleq \eta_{X}(\bar{\Gamma})$ $\left(=\eta_{X, 0}\left(\mathbb{R}^{m}\right)=\eta_{X, 1}\left(\mathbb{R}^{m}\right)\right)$, and assume that $m_{A}+m_{B}>0$, the case $S=0$ being trivial.

For $\gamma_{A} \in \operatorname{supp} A$ and $\gamma_{B} \in \operatorname{supp} B$, let

$$
\left(\gamma_{A} \cdot \gamma_{B}\right)(t) \triangleq \begin{cases}\gamma_{A}(2 t), & t \in[0,1 / 2], \\ \gamma_{B}(2 t-1), & t \in(1 / 2,1] .\end{cases}
$$

Clearly (.) is continuous, hence measurable. Also $\llbracket \gamma_{A} \cdot \gamma_{B} \rrbracket=\llbracket \gamma_{A} \rrbracket+\llbracket \gamma_{B} \rrbracket$. Thus, if we let $\tilde{\eta} \triangleq$ $(\cdot)_{\#}\left(\eta_{A} \times \eta_{B}\right)$, then $\llbracket \tilde{\eta} \rrbracket=m_{B} \llbracket \eta_{A} \rrbracket+m_{A} \llbracket \eta_{B} \rrbracket$. Furthermore, $\tilde{\eta}_{0}=m_{B}(\partial A)^{-}=m_{B}(\partial S)^{-}\left\llcorner\{x\}^{c}\right.$, and $\tilde{\eta}_{1}=m_{A}(\partial B)^{+}=m_{A}(\partial S)^{+}\left\llcorner\{x\}^{c}\right.$, since $\gamma_{A}(1)=\gamma_{B}(0)=x$ a.e. Thus, with

$$
\eta \triangleq \begin{cases}\tilde{\eta} / m_{A}+\left(1-m_{B} / m_{A}\right) \eta_{A}, & m_{A} \geqslant m_{B}, \\ \tilde{\eta} / m_{B}+\left(1-m_{A} / m_{B}\right) \eta_{B}, & m_{A}<m_{B},\end{cases}
$$

we have $\llbracket \eta \rrbracket=\llbracket \eta_{A} \rrbracket+\llbracket \eta_{B} \rrbracket=S, \eta_{1}=(\partial S)^{+}$, and $\eta_{0}=(\partial S)^{-}$. Therefore $\eta$ is a transport measure for $S$. 
Now, as one can see by application of Fubini's theorem,

$$
\begin{aligned}
\alpha_{\tilde{\eta}}(z) & =\iint \chi_{\operatorname{img}\left(\gamma_{A} \cdot \gamma_{B}\right)}(z) \mathrm{d} \eta\left(\gamma_{A}\right) \mathrm{d} \eta\left(\gamma_{B}\right) \leqslant \iint\left(\chi_{\operatorname{img} \gamma_{A}}(z)+\chi_{\operatorname{img} \gamma_{B}}(z)\right) \mathrm{d} \eta\left(\gamma_{A}\right) \mathrm{d} \eta\left(\gamma_{B}\right) \\
& \leqslant m_{B} \alpha_{\eta_{A}}(z)+m_{A} \alpha_{\eta_{B}}(z) .
\end{aligned}
$$

Therefore $\alpha_{\eta}(z)>0$ implies that either $\alpha_{\eta_{A}}(z)>0$ or $\alpha_{\eta_{B}}(z)>0$. We assume the former, the latter case being analogous.

Let then $\Gamma \triangleq\left\{\gamma \in \operatorname{supp} \eta_{A} \mid z \in \operatorname{img} \gamma\right\}$. Define $\tau(\gamma) \triangleq \inf \{t \mid \gamma(t)=z\}$ and $v(\gamma) \triangleq$ $\gamma\llcorner[\tau(\gamma), 1]$ for $\gamma \in \Gamma$. Then $v$ is seen to be measurable by adapting Lemma 2.1. Also let $E \triangleq$ $\llbracket v_{\#}\left(\eta\llcorner\Gamma) \rrbracket\right.$, i.e., $E(\omega)=\int_{\Gamma} \llbracket \gamma\left\llcorner[\tau(\gamma), 1] \rrbracket(\omega) \mathrm{d} \eta_{A}(\gamma)\right.$. Then $\partial E=\alpha_{\eta_{A}}(z)\left(\delta_{x}-\delta_{z}\right)$, because $\gamma(1)=$ $x \eta_{A}$-a.e. and $\alpha_{\eta_{A}}(z)=\eta_{A}(\Gamma)$. Thus we have found an edge between $x$ and $z$. Such edges connect all $z$ with $\alpha_{\eta}(z)>0$ via $x$.

LEMma 3.13 Suppose $S \leqslant T$ is a one-dimensional bundle and $S^{\prime} \leqslant T$ a normal current with $0 \neq-\partial S^{\prime} \leqslant \partial S$. Then $S+S^{\prime}$ contains a cycle if non-zero.

Proof. Since $-\partial S^{\prime} \leqslant \partial S$, there is a density $f=f^{+}+f^{-}$such that $\partial S^{\prime}=-f \partial S\left(\left(\partial S^{\prime}\right)^{\mp}=\right.$ $\left.f^{ \pm}(\partial S)^{ \pm}\right)$. Let $x \in \mathbb{R}^{m}, A=\llbracket \eta_{A} \rrbracket$ and $B=\llbracket \eta_{B} \rrbracket$ be as in Definition 3.4 Define $A^{\prime} \triangleq \llbracket\left(f^{-} \circ p_{0}\right) \eta_{A} \rrbracket$ and $B^{\prime} \triangleq \llbracket\left(f^{+} \circ p_{1}\right) \eta_{B} \rrbracket$. Then

$$
\begin{aligned}
\partial A^{\prime}(\omega) & =\int \llbracket \gamma \rrbracket(\mathrm{d} \omega)\left(f^{-} \circ p_{0}\right)(\gamma) \mathrm{d} \eta_{A}(\gamma) \\
& =\int\left(\delta_{p_{1}(\gamma)}(\omega)-\delta_{p_{0}(\gamma)}(\omega)\right)\left(f^{-} \circ p_{0}\right)(\gamma) \mathrm{d} \eta_{A}(\gamma) \\
& =\int \delta_{x}(\omega)\left(f^{-} \circ p_{0}\right)(\gamma) \mathrm{d} \eta_{A}(\gamma)-\int \delta_{z}(\omega) f^{-}(z) \mathrm{d} \eta_{A, 0}(z) \\
& =\delta_{x}(\omega)\left(f^{-} \eta_{A, 0}\right)\left(\mathbb{R}^{m}\right)-\left(f^{-} \eta_{A, 0}\right)(\omega) \\
& =\left(\partial S^{\prime}\right)^{+}\left(\{x\}^{c}\right) \delta_{x}(\omega)-\left(\partial S^{\prime}\right)^{+}\left\llcorner\{x\}^{c}(\omega) .\right.
\end{aligned}
$$

Likewise,

$$
\partial B^{\prime}(\omega)=-\left(\partial S^{\prime}\right)^{-}\left(\{x\}^{c}\right) \delta_{x}(\omega)+\left(\partial S^{\prime}\right)^{-}\left\llcorner\{x\}^{c}(\omega) .\right.
$$

Thus

$$
\partial A^{\prime}+\partial B^{\prime}+\partial S^{\prime}=\partial S^{\prime}\left(\{x\}^{c}\right) \delta_{x}-\partial S_{\llcorner}^{\prime}\{x\}^{c}+\partial S^{\prime}=0,
$$

where the latter equality follows because $\partial S^{\prime}\left(\{x\}^{c}\right)+\partial S^{\prime}(\{x\})=\partial S^{\prime}\left(\mathbb{R}^{m}\right)=0$ by the compactness of the support of $S^{\prime}$. Finally, we cannot have both $S^{\prime} \leqslant T$ and $-S^{\prime}=A^{\prime}+B^{\prime} \leqslant S \leqslant T$ unless $S^{\prime}=0$, so $A^{\prime}+B^{\prime}+S^{\prime} \neq 0$.

LEMMA 3.14 Suppose $S$ is an acyclic one-dimensional real rectifiable normal current. Then $S=$ $\sum_{i=1}^{\infty} S_{i}$, with each $S_{i}$ a subbundle of $S$.

Proof. We may assume $S \neq 0$. Represent $S=\llbracket \eta \rrbracket$. Let $a_{1}=\operatorname{ess} \sup \alpha_{\eta}$ (in $\mathcal{H}^{1}$ sense). Then $a_{1}>0$ by the rectifiability of $S$. Choose $x$ such that $\alpha_{\eta}(x) \geqslant a_{1}$; such a point exists because $\alpha_{\eta}$ is uppersemicontinuous by [14] Lemma 7.2], and has compact support. Let $\Gamma_{1} \triangleq\{\gamma \in \operatorname{supp} \eta \mid x \in \operatorname{img} \gamma\}$ and $S_{1} \triangleq \llbracket \eta\left\llcorner\Gamma_{1} \rrbracket\right.$. Then $\mathbb{M}\left(\partial S_{1}\right) / 2 \geqslant a_{1}$ and $\partial S_{1} \leqslant \partial S$. Indeed, $S_{1}$ is a subbundle of $S$, as seen by cutting each $\gamma \in \Gamma_{1}$ into two parts at $x$ as follows: Similarly to the proof of Lemma 3.12, define 
$f_{A}(\gamma) \triangleq \gamma\left\llcorner[0, \inf \{t \mid \gamma(t)=x\}]\right.$ and $f_{B}(\gamma) \triangleq \gamma\left\llcorner[\inf \{t \mid \gamma(t)=x\}, 1]\right.$, measurable on $\Gamma_{1}$ by adapting Lemma 2.1 Then let $X=\llbracket\left(f_{X}\right)_{\#}\left(\eta\left\llcorner\Gamma_{1}\right) \rrbracket\right.$ for $X=A, B$.

Next we form $S_{2}$ similarly from $S-S_{1}=\llbracket \eta\left\llcorner\Gamma_{1}^{c} \rrbracket\right.$, and recursively continue the process. Let $S^{\prime} \triangleq S-\sum_{i=1}^{\infty} S_{i}=\llbracket \eta\left\llcorner\left(\bigcup_{i=1}^{\infty} \Gamma_{i}\right)^{c} \rrbracket\right.$. We get ess $\sup \alpha_{\eta\left\llcorner\left(\bigcup \Gamma_{i}\right)^{c}\right.} \leqslant \lim _{i \rightarrow \infty} a_{i}=0$ thanks to the bound $\sum_{i=1}^{\infty} a_{i} \leqslant \mathbb{M}(\partial S) / 2<\infty$ from the construction. Therefore $S^{\prime}=0$, that is, $S=\sum_{i=1}^{\infty} S_{i}$.

LEMma 3.15 Suppose $T$ is an acyclic normal current, $V$ an open set, and $S \leqslant T\llcorner V$ a onedimensional bundle. Then there is a $V$-subbundle $T^{\prime}$ of $T$ with $S \leqslant T^{\prime}$ and $\partial S \wedge \partial T\left\llcorner V \leqslant \partial T^{\prime}\right.$.

Proof. We first consider the case $V=\mathbb{R}^{m}$. Let $I \triangleq \partial S \wedge \partial(S-T)$. Since $I \leqslant \partial S, \partial(S-T)$, there are densities $f \triangleq f^{+}+f^{-}$and $g \triangleq g^{+}+g^{-}$with $f^{ \pm}(x), g^{ \pm}(x) \in[0,1]$ a.e., $I^{ \pm}=f^{ \pm}(\partial S)^{ \pm}$, and $I^{\mp}=g^{ \pm}(\partial(S-T))^{\mp}=g^{ \pm}(\partial(T-S))^{ \pm}$. (Note the signs!) Now,

$$
\begin{aligned}
\partial T & =\partial S+\partial(T-S)=f \partial S+(1-f) \partial S+g \partial(T-S)+(1-g) \partial(T-S) \\
& =I+(1-f) \partial S-I+(1-g) \partial(T-S)=(1-f) \partial S+(1-g) \partial(T-S) .
\end{aligned}
$$

Since a.e. $f^{ \pm} \leqslant f^{ \pm}+\left(1-f^{ \pm}\right)\left(1-g^{\mp}\right)=1-\left(1-f^{ \pm}\right) g^{\mp} \leqslant 1$ and $g^{\mp} \leqslant g^{\mp}+\left(1-f^{ \pm}\right)\left(1-g^{\mp}\right)=$ $1-\left(1-g^{\mp}\right) g^{ \pm} \leqslant 1$, we must have $\left(1-f^{ \pm}\right)\left(1-g^{\mp}\right)=0$ a.e., for otherwise $I^{ \pm}$could not be the maximal subcurrent of both $\partial S$ and $\partial(S-T)$. Therefore, $((1-f) \partial S)^{ \pm} \wedge((1-g) \partial(T-S))^{\mp}=0$, and consequently also

$$
(\partial T)^{ \pm}=\left(1-f^{ \pm}\right)(\partial S)^{ \pm}+\left(1-g^{ \pm}\right)(\partial(T-S))^{ \pm} .
$$

Represent $T-S=\llbracket \eta \rrbracket$, and let $A^{\prime} \triangleq A+\llbracket\left(g^{+} \circ p_{1}\right) \eta \rrbracket$ and $B^{\prime} \triangleq B+\llbracket\left(g^{-} \circ p_{0}\right) \eta \rrbracket$, where $A$ and $B$ are as in Definition 3.4 Then, following the derivation of 3.2 ,

$$
\begin{aligned}
\partial A^{\prime}(\omega) & =\partial A(\omega)+\int \delta_{z}(\omega) g^{+}(z) d \eta_{1}(z)-\int \delta_{p_{0}(\gamma)}(\omega)\left(g^{+} \circ p_{1}\right)(\gamma) \mathrm{d} \eta(\gamma) \\
& =\partial A(\omega)+I^{-}(\omega)-\int \delta_{p_{0}(\cdot)}(\omega)\left(g^{+} \circ p_{1}\right)\left(\left(1-g^{-}\right) \circ p_{0}\right) \mathrm{d} \eta \\
& =\partial A(\omega)+\left(f^{-}(\partial S)^{-}\right)(\omega)-\left(h^{-}(\partial(T-S))^{-}\right)(\omega)
\end{aligned}
$$

for some density $h^{-} \leqslant 1-g^{-}$. The second equality holds because $\left(1-g^{-}\right) \circ p_{0}=1$ a.e. for $\left(g^{+} \circ p_{1}\right) \eta$. Otherwise we would have $\eta\left(\left\{\gamma \mid\left(g^{-} \circ p_{0}\right)(\gamma)\left(g^{+} \circ p_{1}\right)(\gamma)>0\right\}\right)>0$, and then a cycle could be constructed by application of Lemma 3.13 with $S^{\prime} \triangleq \llbracket\left(g^{-} \circ p_{0}\right)\left(g^{+} \circ p_{1}\right) \eta \rrbracket$. The existence of $h^{-}$follows from noting that $((1-g) \partial(T-S))^{-}(\omega)=\int \omega\left(1-g^{-}\right) \mathrm{d} \eta_{0}=$ $\int \delta_{p_{0}(\cdot)}(\omega)\left(\left(1-g^{-}\right) \circ p_{0}\right) \mathrm{d} \eta$.

Similarly to 3.4,

$$
\partial B^{\prime}(\omega)=\partial B(\omega)-\left(f^{+}(\partial S)^{+}\right)(\omega)+\left(h^{+}(\partial(T-S))^{+}\right)(\omega) .
$$

Since $\partial A\left\llcorner\{x\}^{c}=-(\partial S)^{-}\left\llcorner\{x\}^{c}\right.\right.$ and $\partial B\left\llcorner\{x\}^{c}=(\partial S)^{+}\left\llcorner\{x\}^{c}\right.\right.$, we have

$$
\begin{aligned}
& \partial A^{\prime}\left\llcorner\{x\}^{c}=-\left[\left(1-f^{-}\right)(\partial S)^{-}+h^{-}(\partial(T-S))^{-}\right]\left\llcorner\{x\}^{c},\right.\right. \\
& \partial B^{\prime}\left\llcorner\{x\}^{c}=\left[\left(1-f^{+}\right)(\partial S)^{+}+h^{+}(\partial(T-S))^{+}\right]\left\llcorner\{x\}^{c} .\right.\right.
\end{aligned}
$$

Letting $T^{\prime} \triangleq A^{\prime}+B^{\prime}$ and recalling that $\partial A+\partial B=\partial S$, we have

$$
\partial T^{\prime}=(1-f) \partial S+h \partial(T-S), \quad \text { with } h \triangleq h^{+}+h^{-} \geqslant 0 .
$$


Since $h^{ \pm} \leqslant 1-g^{ \pm}$a.e., we see by reasoning similarly to $\sqrt{3.3}$ that $\left(\partial T^{\prime}\right)^{ \pm}=\left(1-f^{ \pm}\right)(\partial S)^{ \pm}+$ $h^{ \pm}(\partial(T-S))^{ \pm}$. Hence $\partial T^{\prime} \leqslant \partial T$. Also, from this property, $\partial A^{\prime}\left\llcorner\{x\}^{c}=-\left(\partial T^{\prime}\right)^{-}\left\llcorner\{x\}^{c}\right.\right.$ and $\partial B^{\prime}\left\llcorner\{x\}^{c}=\left(\partial T^{\prime}\right)^{+}\left\llcorner\{x\}^{c}\right.\right.$. Thus $T^{\prime}$ is a subbundle of $T$ with $S \leqslant T^{\prime}$.

Finally, by (3.3), Lemma 3.3 and the fact

$$
\left(1-g^{ \pm}\right)(\partial(T-S))^{ \pm} \wedge f^{ \pm}(\partial S)^{ \pm}=\left(1-g^{ \pm}\right)(\partial(T-S))^{ \pm} \wedge g^{\mp}(\partial(T-S))^{\mp}=0,
$$

we find $(\partial S \wedge \partial T)^{ \pm}=(\partial S)^{ \pm} \wedge(\partial T)^{ \pm}=\left(1-f^{ \pm}\right)(\partial S)^{ \pm}$. Hence $(\partial S \wedge \partial T)^{ \pm} \leqslant\left(\partial T^{\prime}\right)^{ \pm}$. The case $V=\mathbb{R}^{m}$ has been shown.

If $V \subsetneq \mathbb{R}^{m}$, we replace all the paths in the support of $A^{\prime}-A=\llbracket\left(g^{+} \circ p_{1}\right) \eta \rrbracket$ by the paths only beginning upon entering $V$. This is done by defining the map $v(\gamma) \triangleq \gamma\llcorner[\sup \{t \geqslant 0 \mid$ $\left.\gamma(t) \in V^{c}\right\}$, 1] for $\gamma \in \Gamma_{V} \triangleq\{\gamma \in \operatorname{supp} \eta \mid \gamma(1) \in V\}$. Then $v$ is measurable by adapting Lemma 2.1. For $\left(g^{+} \circ p_{1}\right) v_{\#}\left(\eta\left\llcorner\Gamma_{V}\right)\right.$-a.e. path $\gamma$, img $\gamma \backslash\{\gamma(0)\} \subset V$. We may thus redefine $A^{\prime} \triangleq A+\llbracket\left(g^{+} \circ p_{1}\right) v_{\#}\left(\eta\left\llcorner\Gamma_{V}\right) \rrbracket\right.$. Similarly to (3.4), we then get for some density $h^{-}$, zero outside $V$,

$$
\begin{aligned}
\partial A^{\prime}\llcorner V(\omega) & =\partial A\left\llcorner V(\omega)+\int_{V} \delta_{z}(\omega) g^{+}(z) d \eta_{1}(z)-\int_{\operatorname{img} \gamma \subset V} \delta_{p_{0}(\gamma)}(\omega)\left(g^{+} \circ p_{1}\right)(\gamma) \mathrm{d} \eta(\gamma)\right. \\
& =\partial A\left\llcorner V(\omega)+\left(f^{-}(\partial S)^{-}\llcorner V)(\omega)-\left(h^{-}(\partial(T-S))^{-}\right)(\omega) .\right.\right.
\end{aligned}
$$

We do a similar modification for the paths of $B^{\prime}-B=\llbracket\left(g^{-} \circ p_{0}\right) \eta \rrbracket$, tracing from $t=0$. The entire reasoning above then continues to hold after masking the boundaries with $V$.

Definition $3.5 C$ is a $V$-cycle if $\partial C\llcorner V=0$ and $C\llcorner V \neq 0$.

THEOREM 3.1 Suppose $T$ is a one-dimensional normal current with real rectifiable boundary, and $V$ an open set. Then we have the following equivalences:

(i) If $T$ is edge-connected in $V$, contains no $V$-cycle disjoint in $V$, and $T\llcorner V$ is normal, then $T$ is connected in $V$.

(ii) If $T$ is connected in $V$, acyclic, and real rectifiable, then $T$ is edge-connected in $V$.

Proof. (i) We may assume that $T \neq 0$. To reach a contradiction, suppose therefore that $A$ and $B$ decompose $T$ into non-trivial disjoint components in $V . A$ and $B$ are normal since $T\llcorner V$ was assumed to be. Furthermore, $\partial A\llcorner V, \partial B\llcorner V \leqslant \partial T\llcorner V$ are non-zero, because $T$ was assumed to contain no $V$-cycle disjoint in $V$.

Suppose $E$ is an edge of $T$ in $V$. By the definition of disjointness, the fact supp $E \subset V$, and $V$ being open, we have $\partial(A \wedge E), \partial(B \wedge E) \leqslant \partial E$ and $\partial(A \wedge E)+\partial(B \wedge E)=\partial E$. But $\partial E=\alpha\left(\delta_{x}-\delta_{y}\right)$, whence the compact support of $T$ implies that either $\partial(A \wedge E)=0$ or $\partial(B \wedge E)=0$. We may assume the latter, the other case being analogous. Then, by acyclicity, $B \wedge E=0$, so $E=A \wedge E$, i.e., $E \leqslant A$.

Suppose that $S_{B} \leqslant B$ is normal, and let $S \triangleq E+S_{B}$. Then $\left(\partial E \wedge \partial S_{B}\right)\llcorner V=\partial(A \wedge S) \wedge$ $\partial(B \wedge S)\left\llcorner V=0\right.$, as well as $\partial S_{B}\left\llcorner V \leqslant \partial S\left\llcorner V=\left(\partial S_{B}+\partial E\right)\llcorner V\right.\right.$. Therefore, if $a \in \operatorname{img} \partial E$, then $a \notin \operatorname{img} \partial S_{B}$.

Let then $E_{1}, \ldots, E_{n}$ be a connection of $T$ in $V$ between any $a \in \operatorname{img}(\partial A\llcorner V)$ and $b \in$ $\operatorname{img}(\partial B\llcorner V)$, existing by edge-connectedness and $0 \neq \partial A\llcorner V, \partial B\llcorner V \leqslant \partial T\llcorner V$. By the argument of the previous paragraph (with $S_{B}=B$, and assuming $E=E_{n} \leqslant A$ ), we find $E_{n} \leqslant B$. But since there is always some $x$ with $x \in \operatorname{img} \partial E_{i} \cap \operatorname{img} \partial E_{i+1}$, the argument above (with $S_{B}=E_{i+1}$ ) again tells us that $E_{i} \leqslant B$ for all $i=1, \ldots, n$. But $a \in \operatorname{img} \partial A$ and $a \in \operatorname{img} \partial E_{1}$ is then a contradiction of the same result. Therefore $T$ must be connected in $V$. 
(ii) We say that $x_{0}$ is $V$-bundle-connected to $x_{n}$ if there exists a finite sequence of $V$-subbundles $T_{i}=\llbracket \eta_{i} \rrbracket, i=1, \ldots, n$, with $\alpha_{\eta_{1}}\left(x_{0}\right), \alpha_{\eta_{n}}\left(x_{n}\right)>0$, and either $T_{i} \wedge T_{i+1} \neq 0$, or $\partial T_{i} \wedge \partial T_{i+1}\llcorner V \neq 0$ $(i=1, \ldots, n-1)$. In the former case $\mathcal{H}^{1}\left(\left\{x \in \mathbb{R}^{m} \mid \alpha_{\eta_{i}}(x), \alpha_{\eta_{i+1}}(x)>0\right\}\right)>0$, and in the latter case there exists $x \in \operatorname{img} \partial T\left\llcorner V\right.$ with $\alpha_{\eta_{i}}(x), \alpha_{\eta_{i+1}}(x)>0$. Thus we find that there exist points $x_{i}$ $(i=1, \ldots, n-1)$ with $\alpha_{\eta_{i}}\left(x_{i}\right), \alpha_{\eta_{i+1}}\left(x_{i}\right)>0$. Lemma 3.12 now shows that $x_{0}$ and $x_{n}$ are edgeconnected.

Choose then $x_{0} \in \operatorname{img} \partial T$, and let $\Sigma$ be the set of points $V$-bundled-connected to $x_{0}$. By Lemma 3.12 $T^{\prime}\left\llcorner\Sigma=T^{\prime}\right.$ or $T^{\prime}\left\llcorner\Sigma=0\right.$ for any $V$-subbundle of $T$. Define $T_{A}=T\left\llcorner\Sigma\right.$ and $T_{B}=T\llcorner V-A$. We now intend to show that $T_{A}$ and $T_{B}$ are disjoint components of $T$ in $V$. Since $T\llcorner\Sigma \neq 0$, we must in fact have $T\llcorner\Sigma=T$. This in particular implies that there is a connection between any boundary points of $T$. (Otherwise a different base point $x_{0}^{\prime}$ would yield $\Sigma^{\prime}$ with $\Sigma^{\prime} \cap \Sigma=0$ and $T\left\llcorner\Sigma^{\prime} \neq 0\right.$.)

Clearly $T_{A}$ and $T_{B}$ split any $S \leqslant T\llcorner V$. To show that $\partial S$ is split in $V$, more work is needed. Towards that end, decompose $S$ into subbundles $S_{1}, S_{2}, \ldots$, as shown by Lemma 3.14 . (Since $S=$ $S\left\llcorner V\right.$, the $S_{i}$ are also $V$-subbundles.) According to Lemma 3.15 these bundles can be extended to $V$-subbundles $T_{i}$ of $T$. Consequently, by the construction of $\Sigma, S_{i}\left\llcorner\Sigma=S_{i}\right.$ or 0 . Thus, letting $I \triangleq\left\{i \mid S_{i}\left\llcorner\Sigma=S_{i}\right\}\right.$, we have $T_{A} \wedge S=\sum_{i \in I} S_{i}$ and $T_{B} \wedge S=\sum_{i \notin I} S_{i}$. Therefore also $T_{A} \wedge S$ and $T_{B} \wedge S$ are normal, $\partial\left(T_{A} \wedge S\right)\left\llcorner V, \partial\left(T_{B} \wedge S\right)\left\llcorner V \leqslant \partial S\left\llcorner V\right.\right.\right.$, and $\partial\left(T_{A} \wedge S\right)\left\llcorner V+\partial\left(T_{B} \wedge S\right)\llcorner V=\partial S\llcorner V\right.$.

If $\partial\left(T_{A} \wedge S\right) \wedge \partial\left(T_{B} \wedge S\right)\left\llcorner V \neq 0\right.$, then $\partial S_{i} \wedge \partial S_{j}\llcorner V \neq 0$ for some $i \in I, j \notin I$. There exists a density $h \triangleq h^{+}+h^{-}$with $\left(\partial S_{i} \wedge \partial S_{j}\right)^{ \pm}\left\llcorner V=h^{ \pm}\left(\partial S_{i}\right)^{ \pm}\right.$. We may assume that $h^{-}\left(\partial S_{i}\right)^{-} \neq 0$, the case $h^{+}\left(\partial S_{i}\right)^{+} \neq 0$ being similar. Let $A_{k}=\llbracket \eta_{k} \rrbracket$, and $B_{k}$ be the $A$ and $B$ from Definition 3.4 for $S_{k}, k=i, j$. Denote by $A_{i}^{\prime}=A_{i}+\llbracket \eta^{\prime} \rrbracket$ and $B_{i}^{\prime}$ the corresponding unindexed variables for $S_{i}$ from Lemma 3.15 Let $\hat{A}_{j}=A_{j}+\llbracket\left(h^{-} \circ p_{1}\right) \eta^{\prime} \rrbracket, \hat{B}_{j}=B_{j}$, and $\hat{S}_{j}=\hat{A}_{j}+\hat{B}_{j}$. Finally, extend $\hat{S}_{j}$ as a $V$ subbundle $\hat{T}_{j}$ of $T$ by Lemma 3.15 We must have either $T_{i} \wedge \hat{T}_{j} \neq 0$, or $\left(\partial T_{i}\right)^{-} \wedge\left(\partial \hat{T}_{j}\right)^{-}\llcorner V \neq 0$, both of which imply $j \in I$, since $\hat{T}_{j} \geqslant S_{j}$. The former follows if $\llbracket\left(h^{-} \circ p_{1}\right) \eta^{\prime} \rrbracket \neq 0$, since $\llbracket\left(h^{-} \circ p_{1}\right) \eta^{\prime} \rrbracket \leqslant A_{i}^{\prime}, \hat{A}_{j}$. Otherwise, if $\llbracket\left(h^{-} \circ p_{1}\right) \eta^{\prime} \rrbracket=0$, then $h^{-}\left(\partial S_{i}\right)^{-} \wedge\left(\partial\left(T-S_{i}\right)\right)^{+}=0$, so $h^{-}\left(\partial S_{i}\right)^{-} \leqslant(\partial T)^{-}$. But then $0 \neq h^{-}\left(\partial S_{i}\right)^{-} \leqslant\left(\partial T_{i}\right)^{-}\left\llcorner V,\left(\partial \hat{T}_{j}\right)^{-}\llcorner V\right.$ by Lemma 3.15 We have thus established the contradiction $j \in I$, so $T$ must be edge-connected.

\subsection{The case of finite $V^{c}$}

With the partial equivalence of connectedness and edge-connectedness now at hand, we consider additional properties that follow when $V$ has the form $\left\{x_{1}, \ldots, x_{n}\right\}^{c}$.

Definition 3.6 A collection $\mathcal{A}$ of currents is said to be mutually disjoint in $V$ if for all $A, B \in \mathcal{A}$ with $A \neq B, A$ and $B$ decompose $A+B$ into a pair of disjoint components in $V$.

REMARK 3.6 Any collection of connected components in $V$ of a normal current $T$ is mutually disjoint: Suppose $A_{1} \neq A_{2}$ are connected components of $T$ in $V$. By Lemma 3.5, $A_{1} \vee A_{2}$ is a disjoint component of $T$ in $V$, and then of $A_{i}$ by Remark 3.2. (ii). Thus $A_{1} \wedge A_{2}=0$ and $A_{1}+A_{2}=$ $A_{1} \vee A_{2}$. Since $T\left\llcorner V-A_{1}\right.$ and $A_{1}$ split $A_{1} \vee A_{2}$, the same remark says that $\left(T\left\llcorner V-A_{1}\right) \wedge\left(A_{1} \vee A_{2}\right)=\right.$ $\left(A_{1} \vee A_{2}\right)-A_{1}=A_{2}$ and $A_{1} \wedge\left(A_{1} \vee A_{2}\right)=A_{1}$ are disjoint components of $A_{1}+A_{2}$.

If $T$ is normal, any finite or countable sum of mutually disjoint (disjoint) components of $T$ in $V$ is a disjoint component of $T$ in $V$. This can be seen by repeated application in Definition 3.2 of the equalities

$$
\left(A_{1}+A_{2}\right) \wedge S=\left(A_{1} \wedge S\right)+\left(A_{2} \wedge S\right)=\left(A_{1} \wedge S\right) \vee\left(A_{2} \wedge S\right) \leqslant S, \quad A_{1}, A_{2} \in \mathcal{A},
$$

from Lemmas 3.3 and 3.1 and their boundary and $V$-restricted variants. 
LEMMA 3.16 Suppose $T$ is an acyclic one-dimensional normal current with real rectifiable boundary. Then $T$ is a sum of at most countably many connected components in $V$ whenever $V^{c}$ is a singleton or empty. If $T$ is real rectifiable and contains no circuit, the claim holds whenever $V^{c}$ is finite.

Proof. Any disjoint component $A=A \wedge T$ of $T$ in $V$ is normal (since $T$ is) with $\partial A\llcorner V \leqslant \partial T\llcorner V$, $\partial A\llcorner V+\partial(T-A)\llcorner V=\partial T\llcorner V$, and $\partial A \wedge \partial(T-A)\llcorner V=0$. Therefore, actually $\partial A=\partial T\llcorner U+$ $\sum_{x \in V^{c}} \alpha_{x} \delta_{x}$ for some set $U \subset V$ and $\alpha_{x} \in \mathbb{R}$.

Since $\partial T$ is real rectifiable, there can be at most countably many mutually disjoint components with $\partial A\left\llcorner U \neq 0\right.$. So suppose $\partial A=\sum_{x \in V^{c}} \alpha_{x} \delta_{x}$. If $V^{c}$ is empty, the claim follows by the acyclicity of $T$. If $V^{c}$ is a singleton, and $\partial A=\alpha_{x} \delta_{x}$, compactness of the support implies $\alpha_{x}=0$, and acyclicity implies $A=0$. Thus the claim follows.

If $V^{c}$ is simply finite, any disjoint component with boundary points in $V^{c}$ may still be assumed connected (in $\mathbb{R}^{m}$ !). Now, when $T$ is real rectifiable, there exist edges between the boundary points, by Theorem 3.1 . Since there are only finitely many possible boundary point combinations in $V^{c}$ (although the weights can vary uncountably), a circuit can be constructed if there are uncountably many components of $T$ mutually disjoint in $V$. But $T$ had no circuits.

We have thus shown there are at most countably many disjoint components of $T$, hence at most countably many (mutually disjoint) connected components that sum to $T$.

LEMMA 3.17 Suppose $T$ is a one-dimensional normal current containing no circuit, with real rectifiable boundary. Suppose $C$ and $D$ are disjoint components of $T$ in $V \triangleq\left\{x_{1}, \ldots, x_{n}\right\}^{c}$, edgeconnected in $\mathbb{R}^{m}$. Then for at most one $i \in\{1, \ldots, n\}$, neither $C$ nor $D$ is a disjoint component of $T$ in $V \cup\left\{x_{i}\right\}$.

Proof. Employing the representation $T=\llbracket \eta \rrbracket$, we may observe that for any point $x, \mu_{T}(\{x\})=$ $\int \mathbb{M}\left(\llbracket \gamma \rrbracket\llcorner\{x\}) \mathrm{d} \eta(\gamma)=\int \mathcal{H}^{1}(\operatorname{img} \gamma \cap\{x\}) \mathrm{d} \eta(\gamma)=0\right.$. Therefore, if

$$
\partial(S \wedge C)\left\llcorner\left\{x_{1}\right\}=0 \quad \text { for all normal } S \leqslant T\llcorner V,\right.
$$

then by Lemma 3.8, $C$ is a disjoint component of $T$ in $V^{\prime} \triangleq V \cup\left\{x_{1}\right\}$. Similar conclusions hold when $C$ is replaced by $D$ or $x_{1}$ is replaced by $x_{2}$ in 3.5 . So assume that the condition is violated by all the four combinations.

Let therefore $S$ be in violation of 3.5). We may assume $S=S \wedge C$. If $\partial C\left(x_{1}\right) \neq 0$, we choose $y_{C, 1}=x_{1}$ and let $E_{C, 1}=0$. Otherwise, $\partial(C-S)\left(x_{1}\right)=-\partial S\left(x_{1}\right)$. We may also assume that $\operatorname{img}(\partial S)^{+}=\left\{x_{1}\right\}$ or $\operatorname{img}(\partial S)^{-}=\left\{x_{1}\right\}$ after a possible redefinition restricting a transport measure for $S$ to paths beginning or ending at $x_{1}$. Since the construction for the other case is analogous, we assume for simplicity that actually $\operatorname{img}(\partial S)^{+}=\left\{x_{1}\right\}$. Then $S$ is a bundle, with $A=S$, and $B=0$. We may by Lemma 3.15 extend it to a subbundle $S^{\prime}$ of $C$. In particular, by the proof, $-\partial A^{\prime}\left\llcorner\{x\}^{c} \leqslant(\partial C)^{-}\{x\}^{c}\right.$, while still $\operatorname{img}(\partial A)^{+}=\left\{x_{1}\right\}$. Thus $A^{\prime}$ consists of edges between $x_{1}$ and some points in $\operatorname{img}(\partial C)^{-}, \partial C$ being an at most countable sum of Dirac measures. Choose one of these points as $y_{C, 1}$, and denote the corresponding edge by $E_{C, 1}$.

Similarly to the above, we may produce $E_{C, 2}, E_{D, 1}$, and $E_{D, 2}$, connecting $x_{i}$ to a boundary point $y_{X, i}$ of $X=C, D$ for $i=1,2$. Since $C$ and $D$ are edge-connected we may also find connections between $y_{C, 1}$ and $y_{C, 2}$ in $C$, as well as between $y_{D, 1}$ and $y_{D, 2}$ in $D$. By composing these connections with $E_{C, 1}, E_{C, 2}, E_{D, 1}$, and $E_{D, 2}$, a circuit can be constructed in $T$. This is a contradiction. 


\subsection{Branches}

We now assume that $R \in \mathcal{R}^{*}$, where the latter denotes the set of one-dimensional acyclic normal real rectifiable currents with real rectifiable boundary.

Definition 3.7 Given a point $x \in \mathbb{R}^{m}$, the connected components of $R$ in $\{x\}^{c}$ that are not connected components of $R$ in $\mathbb{R}^{m}$, are called the branches of $R$ at $x$. The point $x$ is called a branch point if the cardinality of branches at $x$ is at least 3 . If the cardinality is at least 2, $x$ is a cut point. If $x$ is not a cut point, it is a non-cut point. (We only apply this last concept to boundary points.)

REMARK 3.7 According to Lemma 3.16, the definition is sound: $R$ is a sum of at most countably many branches. Also, by the proof, all the branches continue to have real rectifiable boundary.

In fact, if $C$ is a branch of $R$ at $x$, then $\partial(S \wedge C)\llcorner\{x\} \neq 0$ for some normal $S \leqslant R$, because otherwise $C$ would be a disjoint component of $R$ in $\mathbb{R}^{m}$ already. If $R$ contains no circuit, then actually $\partial C(x) \neq 0$. Otherwise, similarly to the proof of Lemma 3.17, we could construct edges $E_{1} \leqslant S^{\prime} \leqslant C$ and $E_{2} \leqslant C-S^{\prime}$ connecting $x$ and some $y_{1}, y_{2} \in \operatorname{img} \partial C \backslash\{x\}$, and then form a circuit and a contradiction by connecting these points.

The next two lemmas are intuitively clear, but demand proof due to our abstract current-based definitions of the concepts involved.

LEMMA 3.18 Suppose $R$ is connected, contains no circuit, and $x_{1}$ is a cut point of $R$. Then $x_{2} \neq x_{1}$ is a cut (resp. branch) point of $R$ if and only if it is a cut (resp. branch) point of one of the branches of $R$ at $x_{1}$.

Proof. Let $C_{i}, i=1,2, \ldots$, be the connected components of $R$ in $V \triangleq\left\{x_{1}, x_{2}\right\}^{c}$. Again by Lemma 3.16 there are at most countably many of them. Let $\mathcal{C}_{k}, k=1,2$, be the collection of those $C_{i}$ that remain disjoint components of $R$ in $\left\{x_{k}\right\}^{c}$. Since $C \in \mathcal{C}_{k}$ is connected in $V$, it is connected in $\left\{x_{k}\right\}^{c}$ by Lemma 3.7. Therefore, $C \in \mathcal{C}_{k}$ is a branch of $R$ at $x_{k}$. Since $R$ has no circuit and is real rectifiable, Lemma 3.17 shows that there is at most one index $j$ such that $C_{j} \notin \mathcal{C}_{1} \cup \mathcal{C}_{2}$.

Fix $k \in\{1,2\}$ and let $\ell$ be the other element. Since $R$ is normal, $\sum_{C \in \mathcal{C}_{k}} C$ and $B_{k} \triangleq C_{j}+$ $\sum_{C \in \mathcal{C}_{\ell}} C=R-\sum_{C \in \mathcal{C}_{k}} C$ decompose $R$ into disjoint components in $\left\{x_{k}\right\}^{c} ;$ cf. Remark $3.6 B_{k}$ is also connected: any disjoint component $A$ of $B_{k}$ must be a disjoint component of $R$ in $\left\{x_{k}\right\}^{c}$ by Lemma 3.4 as well as in $V$ by Lemma 3.7 By the latter, $A$ is a sum of $C \in \mathcal{C}_{\ell}$ and $C_{j}$, which contradicts the former unless $A=B_{k}$ or $A=0$. Since $R$ was connected, $B_{k}$ is a branch of $R$ at $x_{k}$, as are $C \in \mathcal{C}_{k}$.

Finally, $C \in \mathcal{C}_{\ell}$ is a branch of $B_{k}$ at $x_{\ell}$, as is $C_{j}$. To see this, first observe that according to the previous paragraph, these are not disjoint components of $B_{k}$ in $\mathbb{R}^{m}$. Secondly, observe that $C=C \wedge B_{k}$ is a disjoint component of $B_{k}$ in $\left\{x_{\ell}\right\}^{c}$, being a disjoint component of $R$ in $\left\{x_{\ell}\right\}^{c}$; cf. Remark 3.2. The same must hold of $C_{j}=B_{k}-\sum_{C \in \mathcal{C}_{\ell}} C$. As these subcurrents are connected in $\left\{x_{\ell}\right\}^{c}$, we have shown the claim: to every branch at $x_{\ell}$ of $R$, there corresponds a branch of $B_{k}$ at $x_{\ell}$, while $B_{k}$ is a branch of $R$ at $x_{k}$.

Lemma 3.19 Suppose $R$ is connected with no circuits and no branch points. Then

(i) At most two points of img $\partial R$ are non-cut points.

(ii) The points of img $\partial R$ can be given a total order $\prec$ satisfying: if $A_{1}$ and $A_{2}$ are the branches of $R$ at a cut point $x \in \operatorname{img} \partial R$, then with either $(i, j)=(1,2)$ or $(i, j)=(2,1)$, we have $y \prec x$ for all $y \in \operatorname{img} \partial A_{i}$, and $x \prec z$ for all $z \in \operatorname{img} \partial A_{j}$. 
Proof. (i) Suppose there are three non-cut points $a, b, c \in \operatorname{img} \partial R$. For each of these points, $x=$ $a, b, c$, there would exist a connection $\mathcal{E}_{x}=\left\{\left(\tau_{x, i}, E_{x, i}\right)\right\}_{i=1}^{n_{x}}$ in $\left\{x^{c}\right\}$ between the other two points. Here $\tau_{x, i}= \pm 1$ is the direction of the edge $E_{x, i}$. We may assume all the connections to have equal strength. We claim that $\mathcal{E} \triangleq \mathcal{E}_{a} \cup \mathcal{E}_{b} \cup \mathcal{E}_{c}$ would form a circuit in $R$.

By rectifiability and the finite number of edges in $\mathcal{E}$, we may, similarly to the proof of Lemma 3.11. assume that any edges with a non-zero intersection are equal, and that each edge occurs at most once in each of the connections $\mathcal{E}_{x}, x=a, b, c$. Note that $\sum_{(\tau, E) \in \mathcal{E}} \tau \partial E=0$. Therefore, if the edges do not form a circuit, $\sum_{E \in \mathcal{E}} \tau_{E} E=0$. Thus, positively weighted edges correspond exactly to negatively weighted edges: each edge occurs exactly twice; once in two of the three connections, with different direction.

Now, take, e.g., $x=a$. If there are two distinct edges in $\mathcal{E}$ (disregarding direction) with $x$ as a boundary point, then set $w=x$. Otherwise, disregard both copies of the sole edge $E$ with $x$ as a boundary point, and continue with $x$ as the other boundary point of $E$. By repeating the process, eventually distinct edges and $w \neq b, c$ must be found, to reach both $b$ and $c$. Now, some of the remaining edges of $\mathcal{E}$ form a connection between $b$ and $c$ and from $a$, passing through $w$. Therefore, if there exists a connection between any two of the points $a, b, c$ in $\{w\}^{c}$, a circuit is found. If this does not happen, there must exist at least two connected components of $R$ in $\{w\}^{c}$, i.e., $w$ is a cut point in contradiction to our assumptions.

(ii) If there are only two points in img $\partial R$, they must both be non-cut points, and can be ordered arbitrarily.

Assume then that there are more than two points, and exactly one non-cut point $x_{0} \in \operatorname{img} \partial R$. Let $x \in \operatorname{img} \partial R$ be a cut point, with $A$ and $B$ the branches of $R$ at $x$. Since $\partial A\left\llcorner\{x\}^{c}\right.$ and $\partial B\left\llcorner\{x\}^{c}\right.$ split $\partial R$, we may assume that $x_{0} \in \operatorname{img} \partial A$, possibly after replacing the roles of $A$ and $B$. We then declare $y \prec x$ for all $y \in \operatorname{img} \partial A$.

Suppose that $y \neq x, x_{0}$. By Lemma 3.18, $y$ is a cut boundary point of $A$. Let $A_{y}$ and $B_{y}$ be the branches of $A$ at $y$, ordered so that $x_{0} \in \operatorname{img} \partial A_{y}$ and $x \in \operatorname{img} \partial B_{y}$. This is doable by (i), as $x$ and $x_{0}$ remain non-cut boundary points of either $A_{y}$ or $B_{y}$, and $y$ is by definition a non-cut boundary point of both. Now note that $B_{y}+B$ is edge-connected (via $x$ ), so $A_{y} \leqslant A$ and $B_{y}+B$ are the branches of $R$ at $y \prec x$. This shows antisymmetry and transitivity of the relation $\prec$. Totality follows analogously by considering $y \in \operatorname{img} \partial B$.

If there is another non-cut boundary point $x_{1}$ of $R$, we set $x \prec x_{1}$ for all $x \in \operatorname{img} \partial R$, noting that we cannot have $x_{1} \in \operatorname{img} \partial A$, i.e., $x_{1} \prec x$, for any $x \in \operatorname{img} \partial R \backslash\left\{x_{1}\right\}$. For, otherwise all $x_{0}, x_{1}$, and $x$ would be non-cut boundary points of $A$, in contradiction to (i)

Finally, if there are no non-cut boundary points of $R$, we take some cut boundary point, apply the above process to the halves obtained that way, and combine the results, reversing the order on the other half.

\section{Optimal networks}

\subsection{The problem}

Given a signed measure $\mu=\mu^{+}-\mu^{-}$with compact support and $\mu^{+}\left(\mathbb{R}^{m}\right)=\mu^{-}\left(\mathbb{R}^{m}\right)<\infty$, we consider the problem

$$
\min _{\partial(W+R)=\mu} J(W, R), \quad J(W, R) \triangleq J_{W}(W)+J_{R}(R),
$$

where $W$ and $R$ are one-dimensional real flat chains of finite mass. We assume that $J_{W}$ is 
subadditive:

$$
J_{W}\left(W_{1}+W_{2}\right) \leqslant J_{W}\left(W_{1}\right)+J_{W}\left(W_{2}\right)
$$

and $J_{R}$ is strictly "quasi-concave on circuits":

$$
\min \left\{J_{R}\left(R+C_{1}-C_{2}\right), J_{R}\left(R-C_{1}+C_{2}\right)\right\}<J_{R}(R)
$$

whenever $\left(C_{1}, C_{2}\right)$ is a circuit of $R$. To ensure a (real) rectifiable set of stations, we also assume

$$
J_{R}(R)<\infty \text { implies that } \partial R \text { is real rectifiable and } R \text { normal. }
$$

Then both $W$ and $R$ are actually normal currents. In fact, $R$ is real rectifiable and acyclic, i.e., $R \in \mathcal{R}^{*}$, as shown by Lemma 4.1 below.

By further assumptions on $J_{R}$, our plan is to show that the number of stations is actually finite, and consequently $R$ is a Lipschitz image of a polyhedral current. These assumptions take the form

$$
J_{W}(B)+J_{R}(R-B)<J_{R}(R) \quad \text { when } B \in \mathcal{B},
$$

for a varying set $\mathcal{B}$. Combining with $\mathrm{A}-\mathrm{S}$ ) it then follows that $J(W+B, R-B)<J(W, R)$, so that decrease is achieved while maintaining the constraint $\partial(W+R)=\mu$.

\subsection{Circuits and rectifiability}

The pair $(W, R)$ of finite mass real flat chains is said to be finitely optimal if it solves $(\mathbb{P})$ and $J(W, R)<\infty$. The same is said of just $R$ if this holds with some $W$.

Lemma 4.1 Any finitely optimal $R$ contains no circuit and is real rectifiable. Thus $R \in \mathcal{R}^{*}$.

Proof. Clearly $[\mathrm{A}-\mathrm{C}]$ and optimality exclude circuits, since $\partial R^{\prime}=\partial R$ if $R^{\prime} \triangleq R \pm\left(C_{1}-C_{2}\right)$ and $\left(C_{1}, C_{2}\right)$ is a circuit of $R$.

Since $R$ contains no circuit, it is also acyclic (choose $C_{2}=0$ ). Therefore, we may use the representation (2.2) by transport measures, $R=\llbracket \eta \rrbracket$.

By $\widehat{\mathrm{A}-\mathrm{N}]}, \partial R=\sum_{i=1}^{\infty} \alpha_{i} \delta_{x_{i}}$ for some $\alpha_{i} \in \mathbb{R}$ and $x_{i} \in \mathbb{R}^{m}$. Therefore, for some pairs $(i, j)$, there is a set $\Gamma^{i j} \subset \operatorname{supp} \eta$ of non-zero $\eta$-measure of transport paths between $x_{i}$ and $x_{j}$. According to Lemma 3.10, $\eta\left\llcorner\Gamma^{i j}\right.$ is concentrated on a singleton $\left\{\gamma^{i j}\right\}$. Because there are at most countably many paths $\gamma^{i j}$ (modulo equivalent parametrisations) corresponding to different pairs $(i, j), R$ must be real rectifiable.

REMARK 4.1 Even if $R=\llbracket \eta \rrbracket$ is real rectifiable with real rectifiable boundary, $\eta$ may not be concentrated on a countable set if $R$ contains a circuit. Just consider the acyclic current on the sides of the unit rectangle, with boundary points of mass \pm 1 at some of the two opposing corners. If copies scaled to $2^{-i}(i=1,2, \ldots)$ of the side length (but preserving boundary mass) are chained together at their opposing corners, one obtains a normal real rectifiable current with two boundary points that have $2^{\mathbb{N}}$, i.e., uncountably many possible paths between them.

\subsection{Connected components}

LEMma 4.2 Suppose A-D holds for some $\beta>0$ with

$$
\mathcal{B}_{\text {disj }}^{\beta} \triangleq\{B \text { is a disjoint component of } R \text { with } \mathbb{M}(\partial B) \leqslant \beta\} .
$$

Then any finitely optimal $R$ consists of finitely many connected components. 
Proof. By Lemma 3.16, $R$ is the sum of at most countably many connected components $\left\{R_{i}\right\}_{i=1}^{\infty}$. Since $\sum_{i=1}^{n} \partial R_{i} \subset \partial R$ for all finite subcollections, we have

$$
\begin{aligned}
\sum_{i=1}^{n} \mathbb{M}\left(\partial R_{i}\right) & \leqslant \sum_{i=1}^{n}\left(\mathbb{M}(\partial R)-\mathbb{M}\left(\partial R-\partial R_{i}\right)\right) \\
& \leqslant \mathbb{M}(n \partial R)-\mathbb{M}\left(n \partial R-\sum_{i} \partial R_{i}\right) \leqslant \mathbb{M}\left(\sum_{i} \partial R_{i}\right) \leqslant \mathbb{M}(\partial R)
\end{aligned}
$$

Thus $\lim \sup _{i \rightarrow \infty} \mathbb{M}\left(\partial R_{i}\right)=0$. Consequently $\mathbb{M}\left(\partial R_{i}\right)<\beta$ for suitably large $i$, and an application of A-D yields a contradiction to optimality.

\subsection{Branching}

LEMMA 4.3 Suppose A-D holds for some $\beta>0$ with

$$
\mathcal{B}_{\mathrm{disj}, \mathrm{x}}^{\beta} \triangleq\left\{B \text { is a disjoint component of } R \text { in }\{x\}^{c} \text { with } \mathbb{M}(\partial B) \leqslant \beta \mid x \in \mathbb{R}\right\} .
$$

If $(W, R)$ is optimal and $J(W, R)<\infty$, then each connected component of $R$ has finitely many branch points, each with finitely many branches.

Proof. Let $T$ be a connected component of $R$. Let us denote the branch points of $T$ by $X$. Choose some $x \in X$, and let $\left\{T_{i}\right\}_{i \in I}$ be the branches of $T$ at $x$. Set $X^{\prime}=\{x\}$. Then proceed recursively as follows.

Let $X^{\prime}\left(T_{i}\right) \subset X^{\prime}$ denote the smallest subset such that $T_{i}$ remains a disjoint component of $T$ in $\left(X^{\prime}\left(T_{i}\right)\right)^{c}$. Such a set exists, for if there are two contenders, finiteness of the sets $\left(\mu_{T}\left(X^{\prime}\right)=0\right)$ and the definition of disjointness establishes that the intersection satisfies the requirements of Definition 3.2 as well. Let $I_{x} \subset I$ denote the indices $i$ such that $X^{\prime}\left(T_{i}\right)=\{x\}$, i.e., $T_{i}$ is a branch of $T$ at $x$ (as is the case on the first iteration). If for some $i \in I_{x}$ we had $\mathbb{M}\left(\partial T_{i}\right)<\beta$, an application of (A-D) to $B=T_{i}$ would show that the cost $J$ can be reduced. Therefore $\mathbb{M}\left(\partial T_{i}\right) \geqslant \beta$ for all $i \in I_{x}$. But $\sum_{i \in I_{x}} \mathbb{M}\left(\partial T_{i}\right) \leqslant \sum_{i \in I_{x}} 2 \mathbb{M}\left(\partial T_{i}\left\llcorner\{x\}^{c}\right) \leqslant 2 \mathbb{M}\left(\partial T\left\llcorner\{x\}^{c}\right) \leqslant 2 \mathbb{M}(\partial R)<\infty\right.\right.$. This implies that $I_{x}$ is finite.

Note that according to Lemma 3.17, since $R$ contains no circuit by Lemma 4.1, if $x^{\prime} \in X^{\prime} \backslash\{x\}$, then $x^{\prime} \in X^{\prime}\left(T_{j}\right)$ for at most one branch. Since $X^{\prime}$ is finite, there can thus only be finitely many branches $T_{i}, i \in I \backslash I_{x}$, that are not branches of $T$ at $x$. Thus $I$ is finite, which shows the second half of the claim of the lemma. For the first half, assume to the contrary that $X$ is infinite. Then one of the branches, say $T_{j}$, continues to have infinitely many points of $X \backslash X^{\prime}$ as its branch points, by Lemma 3.18 .

Update $\tilde{T} \triangleq T_{j}$, and let $\tilde{X}$ be the branch points of $T$ that remain branch points of $\tilde{T}$. Choose a new point $\tilde{x} \in \tilde{X} \backslash X^{\prime}$, and update also $\tilde{X}^{\prime} \triangleq X^{\prime}(\tilde{T}) \cup\{\tilde{x}\}$.

If there is at least one branch $T_{i}$ distinct from $\tilde{T}$, such that $X^{\prime}\left(T_{i}\right)=\{x\}$, then $\mathbb{M}(S) \geqslant \beta / 2$ for $S \triangleq \partial T_{i}\left\llcorner\{x\}^{c} \leqslant \partial T \leqslant \partial R\right.$. Therefore, by iterating the above arguments with $\left(T, X, X^{\prime}, x\right) \triangleq$ $\left(\tilde{T}, \tilde{X}, \tilde{X}^{\prime}, \tilde{x}\right)$, eventually a discardable low-mass branch must be found, violating the optimality of $(W, R)$.

If it however happens that all the other branches have $X^{\prime}\left(T_{i}\right) \supsetneq\{x\}$, then $\# \tilde{X}^{\prime}<\# X^{\prime}$, as at least two points of $X^{\prime}$ are not contained in $X^{\prime}(\tilde{T})$. This is due to Lemma 3.17 and $x$ being a branch point of $T$, whence there are at least two branches other than $\tilde{T}$. Consequently, since $X^{\prime}$ is finite, this case can only happen finitely many times in uninterrupted sequence. 
LEMmA 4.4 Let $B \triangleq\left\{x \in \mathbb{R}^{m} \mid x\right.$ is a branch point of $\left.R\right\}$. Then, under the assumptions of Lemma 4.3 , every connected component of $R$ in $B^{c}$ has exactly two non-cut boundary points and no branch points.

Proof. Let $C$ be one of the connected components of $R$ in $B^{c}$. It has no circuits, by Lemma 4.1, and no branch points, by Lemma 3.18. Therefore, by Lemma 3.19(i), $C$ has at most two non-cut boundary points. If $C$ has finitely many cut boundary points, it must have exactly two non-cut boundary points. To see this, cut $C$ into two halves at one of its cut boundary points $x$. If $C$ has fewer than two non-cut boundary points, one of the halves will have at most $x$ as a non-cut boundary point. Recursively continuing the process on this half until all the finitely many cut boundary points have been exhausted (recall Lemma 3.18) will yield a current with fewer than two boundary points. This is not possible by acyclicity and compact support.

Suppose then that $C$ has countably many cut boundary points. Every $x \in B$ is a non-cut point, so at most two of them can be boundary points. If we denote this set by $B_{C}$, then $C$ is a disjoint component of $R$ in $\left(B_{C}\right)^{c}$. If $B_{C}$ has at least two elements, we are done, so assume that it has fewer than two elements.

If $y$ is a cut boundary point of $C$, then one of the branches, $A$, of $C$ at $y$ will be a disjoint component of $R$ in $\{y\}^{c}$ by Lemma 3.17. If $\mathbb{M}(\partial A)<\beta$, then according to (A-D), cutting away $A$ would improve cost. To reach a contradiction, it remains to choose $y$ appropriately. This can be done by ordering the cut boundary points in a sequence $y_{1}, y_{2}, \ldots$, and choosing $K$ to achieve $\sum_{i=K+1}^{\infty}\left|C\left(y_{k}\right)\right|<\beta$. Then we take $y \in Y \triangleq\left\{y_{1}, \ldots, y_{K}\right\}$ to be a minimal or maximal point of $Y \cup B_{C}$, in the order of Lemma 3.19 (ii)

\subsection{Finiteness}

We finally show that there are actually only finitely many stations under certain assumptions.

LEMMA 4.5 Suppose A-D holds in either of the cases:

(i) For a given $\delta>0$, with some $g:(0, \infty) \rightarrow(0, \infty)$ and

$$
\mathcal{B}_{\text {seg }}^{\delta, g}=\{\partial R(y) \llbracket x, y \rrbracket|x, y \in \operatorname{img} \partial R ; y \in B(x, \delta) ;| \partial R(y) \mid \leqslant g(|\partial R(x)|)\} .
$$

(ii) With $\mathcal{B}_{\mathrm{disj}, \mathrm{x}}^{\beta}$ for some $\beta>0$, and for some $g:(0, \infty) \rightarrow(0, \infty)$ and given $M, \theta>0$ with

$$
\mathcal{B}_{\mathrm{conn}}^{M, \theta, g}=\left\{\begin{array}{l|l}
\partial R(y) C & \begin{array}{l}
x, y \in \operatorname{img} \partial R ; \theta C_{1}, \theta C_{2} \leqslant R ; C \triangleq C_{1}-C_{2} \\
\partial C=\delta_{y}-\delta_{x} ; \mathbb{M}(C) \leqslant M ;|\partial R(y)| \leqslant g(|\partial R(x)|)
\end{array}
\end{array}\right\}
$$

Then the set img $\partial R$ is finite if $(W, R)$ is finitely optimal.

Proof. Write $m_{x} \triangleq \partial R(x)$. Under the assumption that $R$ and then $\partial R$ are supported on a compact set, the claims follow in both cases by showing that in a suitable fixed neighbourhood of each station $m_{x} \delta_{x}$, there can be only finitely many other stations $m_{y} \delta_{y}$. This in turn follows from showing that there is a lower limit on $\left|m_{y}\right|$ in this neighbourhood, since there can be only finitely many stations of greater mass.

(i) In this case, we may simply lay new track to fuse nearby stations. Given $\delta>0$, finitely many balls $B(x, \delta)$ with $x \in \operatorname{img} \partial R$ cover img $\partial R$. So suppose $y \in B(x, \delta)$ with $\left|m_{y}\right| \leqslant g\left(\left|m_{x}\right|\right)$. Then, by (A-D) and our assumptions, $J\left(W+m_{y} \llbracket x, y \rrbracket, R-m_{y} \llbracket x, y \rrbracket\right)<J(W, R)$, yielding a contradiction to optimality. 
(ii) Note that $\mathrm{A}-\mathrm{D}$ holding for $\mathcal{B}_{\mathrm{disj}}$ follows from it holding for $\mathcal{B}_{\mathrm{disj}, \mathrm{x}}$, thanks to the compact support of $R$. Therefore, $R$ has finitely many connected components (in $\mathbb{R}^{m}$ ) by Lemma 4.2. By Lemma 4.3 , each of them has finitely many branch points with finitely many branches. Therefore, if $\left\{R_{i}\right\}_{i=1}^{\infty}$ are the connected components of $R$ in $B^{c}$, then at most finitely many of them are disjoint in $\mathbb{R}^{m}$ or $\{x\}^{c}$ for $x$ in $B$, the set of branch points of $R$. An application of Lemma 3.17 then shows that $R$ can actually have only finitely many connected components $R_{1}, \ldots, R_{N}$ in $B^{c}$. By Lemma 4.4, each $R_{i}$ has no branch points, and exactly two non-cut boundary points $x_{i, 0}$ and $x_{i, 1}$.

Fix $i$, and let $E_{1}, \ldots, E_{n} \leqslant R_{i}$ be the connection between $x_{i, 0}$ and $x_{i, 1}$. Denote its strength by $\theta_{i}$, and the traversal directions of the edges by $\tau_{j}= \pm 1$.

Let $x$ and $y$ be distinct cut boundary points of $R_{i}$. By Lemma 3.18 $R_{i}$ decomposes into three connected components $A, B_{0}$ and $B_{1}$ in $\{x, y\}^{c}$, with $A$ the unique one that is not a disjoint in $\{x\}^{c}$ or $\{y\}^{c}$, and $x_{i, j} \in \operatorname{img} \partial B_{j}, j=0,1$. Then $E_{j}^{\prime} \triangleq A \wedge E_{j}$ is a (possibly zero) edge in $A$. We cannot have $E_{i}^{\prime}=0$ for all $i$, because otherwise a circuit could be found by combining $E_{1}, \ldots, E_{n} \leqslant B_{0}+B_{1}$ with connections of suitable pairs of $x, y, x_{i, 0}$, and $x_{i, 1}$ in $A, B_{1}$, and $B_{2}$. Likewise, we cannot have $E_{i} \wedge B_{j}=0$ for all $i$ for any $j=0,1$. Therefore, since $x$ or $y$ is in img $\partial E_{j}^{\prime}$ whenever $0 \neq E_{j}^{\prime} \neq E_{j}$, and the edges $E_{j}^{\prime}$ cannot form a circuit, we must have $x \in \operatorname{img} \partial E_{i}^{\prime}$ and $y \in \operatorname{img} \partial E_{j}^{\prime}$ for some $i, j \in\{1, \ldots, n\}$. Thus $E_{1}^{\prime}, \ldots, E_{n}^{\prime}$ must form a connection between $x$ and $y$.

Let $C_{1}=\sum_{\tau_{j}= \pm 1} E_{j}^{\prime} / \theta_{i}, C_{2}=\sum_{\tau_{j}=\mp 1} E_{j}^{\prime} / \theta_{i}$, and $C=C_{1}-C_{2}$, with the directions/signs summed over chosen in the order that achieves $\partial C=\delta_{y}-\delta_{x}$. Then $\mathbb{M}(C) \leqslant M_{i} \triangleq \sum_{j=1}^{n} \mathbb{M}\left(E_{j}\right) / \theta_{i}$, while $M_{i} \leqslant M \triangleq \max _{i=1, \ldots, N} M_{i}$, and $\theta_{i} \geqslant \theta \triangleq \min _{i=1, \ldots, N} \theta_{i}$. But then, by (A-D) and our assumptions, we have the contradiction $J\left(W+m_{y} C, R-m_{y} C\right)<J(W, R)$, provided $\left|m_{y}\right|<$ $g\left(\left|m_{x}\right|\right)$.

The results of this section are summarised in the following theorem. To actually show the existence of a solution, it is more convenient to work with more specific objective functions. This is done in the next section.

ThEOREM 4.1 Suppose the assumptions $(\mathrm{A}-\mathrm{S}), \mathrm{A}-\mathrm{C})$, and $(\mathrm{A}-\mathrm{N})$ hold and that $\mathrm{A}-\mathrm{D}$ ) holds with

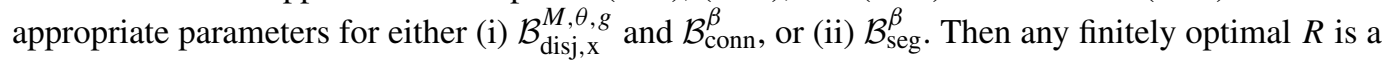
Lipschitz image of a polyhedral current with no circuit.

Proof. By combining the above lemmas, $R$ is real rectifiable, and has no circuit, while $\partial R$ is finite. By considering $R=\llbracket \eta \rrbracket=\sum_{i=1}^{s} \sum_{j=1}^{d} \alpha_{i j} \llbracket \gamma_{i j} \rrbracket$, we find a finite collection of Lipschitz paths on which $R$ is concentrated. This says that $R$ is a Lipschitz image of a polyhedral current.

\section{Weighted areas}

\subsection{Definitions and basic results}

Let $\mathcal{W}$ denote the set of non-decreasing concave functions $[0, \infty) \rightarrow[0, \infty)$ that take the value 0 at the origin. Such functions are subadditive as well. We symmetrically extend them to all of $\mathbb{R}$ for convenience of notation. Throughout the section, $w, r, s \in \mathcal{W}$. We write $w \preccurlyeq r$ if $r \neq 0$ and for some $\tau>0, w / r$ is a non-decreasing function in $(0, \tau)$ (and thus has a limit from the right at zero). If actually $\lim _{t \searrow 0} w(t) / r(t)=0$, we write $w \prec r$.

The identity function is denoted by id : $t \mapsto t$. Note that id $\preccurlyeq r$ with $\tau=\infty$ for all $r \in \mathcal{W}$ not identically zero (as follows from $0 \leqslant r^{\prime}(t) \leqslant r(t) / t$ and $r(s)-r(t) \leqslant r^{\prime}(t)(s-t), s \geqslant t$ ). Other 
important examples of functions in $\mathcal{W}$ include $t \mapsto t^{\alpha}$ for $\alpha \in[0,1)$ (with the convention $0^{0}=0$ ). If $\alpha<\beta$, then $t^{\beta} \prec t^{\alpha}$ with $\tau=\infty$.

Given a weight function $r \in \mathcal{W}$, we define the $r$-mass of a $k$-dimensional real rectifiable current $T=\llbracket \Sigma \rrbracket\llcorner\theta$ as

$$
\mathbb{M}^{r}(T) \triangleq \int_{\Sigma} r(\theta) \mathrm{d} \mathcal{H}^{k}
$$

$\mathbb{M}^{r}$ is lower-semicontinuous on real rectifiable currents with regard to convergence in the Whitney flat distance $\mathbb{F}$ (as follows similarly to the proof for (integer) rectifiable currents by De Pauw and Hardt [10] according to Paolini and Stepanov [14]). It may therefore be extended to a lowersemicontinuous function on the set of flat chains by taking the infimum of $\liminf \mathbb{M}^{r}\left(T_{i}\right)$ over all approximating sequences. According to White [15, 6], one could equally well use polyhedral currents in the approximating sequence. Clearly $\mathbb{M}^{r}$ is also subadditive for real rectifiable $T_{1}$ and $T_{2}$, i.e., $\mathbb{M}^{r}\left(T_{1}+T_{2}\right) \leqslant \mathbb{M}^{r}\left(T_{1}\right)+\mathbb{M}^{r}\left(T_{2}\right)$, and otherwise the property follows by approximation.

LEMma 5.1 Suppose id $\prec r$ and $T$ is a flat chain with $\mathbb{M}(T)+\mathbb{M}^{r}(T)<\infty$. Then $T$ is real rectifiable. If $T$ is 0 -dimensional and $r(\infty-) \triangleq \lim _{t \rightarrow \infty} r(t)=\infty$, then automatically $\mathbb{M}(T) \leqslant$ $r^{-1}\left(\mathbb{M}^{r}(T)\right)$, so it suffices to have $\mathbb{M}^{r}(T)<\infty$.

Proof. Since $r$ increases at 0 faster than any linear function, there is no continuous non-constant curve of finite length when the distance between two points is given by $(x, y) \mapsto(\mathrm{id}+r)(\|x-y\|)$. Thus the results of White [16, 8] show the rectifiability of $T$.

In the case that $T$ is 0 -dimensional with $\mathbb{M}^{r}(T)<\infty$, approximate $T$ in $\mathbb{F}$ by real rectifiable currents $T_{k}=\sum_{i=1}^{n_{k}} \alpha_{k, i} \delta_{x_{k, i}}$ with $\mathbb{M}^{r}\left(T_{k}\right) \rightarrow \mathbb{M}^{r}(T)$. Then $r\left(\mathbb{M}\left(T_{k}\right)\right)=r\left(\sum_{i}\left|\alpha_{k, i}\right|\right) \leqslant$ $\sum_{i} r\left(\alpha_{k, i}\right)=\mathbb{M}^{r}\left(T_{k}\right)$. But $\mathbb{M}(T) \leqslant \liminf \mathbb{M}\left(T_{k}\right)$ and $r$ is continuous and non-decreasing, whence $\mathbb{M}(T) \leqslant r^{-1}\left(\mathbb{M}^{r}(T)\right)<\infty$.

The next two lemmas adapt results of [14].

LEMMA 5.2 Suppose $T$ is a real rectifiable current with $\mathbb{M}(T)<\infty$. Let $F(\sigma) \triangleq \mathbb{M}^{r}(T\llcorner\sigma)$ for $\sigma \in K \triangleq\left\{\sigma: \mathbb{R}^{m} \rightarrow[0, \infty) \mid \sigma\right.$ Borel $\}$. Then $F$ is concave on $K$.

In particular, if $\tilde{\sigma}: \mathbb{R}^{m} \rightarrow[-1,1]$ is Borel, then $f(t) \triangleq F(1+t \tilde{\sigma})$ is concave for $t \in[-1,1]$, strictly if $T\llcorner\tilde{\sigma} \neq 0$ and $r$ is strictly concave.

Proof. Since $T=\llbracket \Sigma \rrbracket\llcorner\theta$ and $T\llcorner\sigma=\llbracket \Sigma \rrbracket\llcorner(\sigma \theta)$, we get

$$
\begin{aligned}
\lambda F(\sigma)+(1-\lambda) F\left(\sigma^{\prime}\right) & =\int_{\Sigma}\left(\lambda r(\sigma \theta)+(1-\lambda) r\left(\sigma^{\prime} \theta\right)\right) \mathrm{d} \mathcal{H}^{k} \\
& \leqslant \int_{\Sigma} r\left(\left(\lambda \sigma+(1-\lambda) \sigma^{\prime}\right) \theta\right) \mathrm{d} \mathcal{H}^{k}=F\left(\lambda \sigma+(1-\lambda) \sigma^{\prime}\right) .
\end{aligned}
$$

Clearly, if $r$ is strictly concave, then the inequality is strict for $\lambda \in(0,1)$ unless $\theta \sigma=\theta \sigma^{\prime} \mathcal{H}^{k}\llcorner\Sigma$ a.e. In the case of $f$ this means $\tilde{\sigma}=0$ a.e. for $\mu_{T}=\theta \mathcal{H}^{k}\llcorner\Sigma$, so the claim follows.

LEMMA 5.3 Suppose $T$ is an acyclic one-dimensional real rectifiable normal current. If $w \preccurlyeq r$ with non-decrease threshold $\tau$, and $\mathbb{M}(\partial T) / 2 \leqslant \tau$, then

$$
\mathbb{M}^{w}(T) \leqslant \mathbb{M}^{r}(T)\left[\frac{w}{r}(\mathbb{M}(\partial T) / 2)\right] .
$$

If $\tau=\infty$ and $(w / r)(\infty-)=\infty$, then it suffices to assume that $T$ is an acyclic one-dimensional real rectifiable current of finite mass and compact support. 
Proof. Clearly, if $(w / r)(\infty-)=\infty$, then (5.1) holds whenever $\mathbb{M}(\partial T)=\infty$, so we may assume $\mathbb{M}(\partial T)<\infty=\tau$, and the special case reduces to the generic one. We may also assume that $\mathbb{M}(\partial T)=0$, as otherwise $T=0$, and there is nothing to prove.

We have the expression $T=\llbracket \Sigma \rrbracket\llcorner\theta$, where $\Sigma$ is a rectifiable set. According to 14 , Lemma 7.1 \& Theorem 7.3], $\theta \leqslant \mathbb{M}(\partial T) / 2 \mathcal{H}^{1}$-a.e. Thus, because $\mathbb{M}(\partial T) / 2 \leqslant \tau$ by assumption, we find

$$
\mathbb{M}^{w}(T)=\int_{\Sigma} w(\theta) \mathrm{d} \mathcal{H}^{1} \leqslant \int_{\Sigma} \frac{w(\mathbb{M}(\partial T) / 2)}{r(\mathbb{M}(\partial T) / 2)} r(\theta) \mathrm{d} \mathcal{H}^{1}=\mathbb{M}^{r}(T)\left[\frac{w}{r}(\mathbb{M}(\partial T) / 2)\right] .
$$

The next lemma provides a variant of standard approximation results, employing $\mathbb{M}^{r}(T)$ instead of $\mathbb{M}(T)$, and with preservation of rectifiable boundary.

LEMmA 5.4 Suppose $0<r^{\prime}(0)<\infty, T$ is a one-dimensional real flat chain with compact support, and $\mathbb{M}^{r}(T)+\mathbb{M}(\partial T)<\infty$. Then, given $\epsilon>0$, there exists a normal real rectifiable current $T^{\prime}$ with real rectifiable boundary, supp $T^{\prime} \subset$ conv supp $T+B(0, \epsilon), \mathbb{M}\left(\partial T^{\prime}\right) \leqslant \mathbb{M}(\partial T)+\epsilon, \mathbb{M}^{r}\left(T^{\prime}\right) \leqslant$ $\mathbb{M}^{r}(T)+\epsilon$, and $\mathbb{F}\left(T^{\prime}-T\right) \leqslant \epsilon / r^{\prime}(0)$.

If $\phi \leqslant \partial T$ is real rectifiable, then we can take $\phi \leqslant \partial T^{\prime}$. In particular, if $\partial T$ is real rectifiable, then $\partial T^{\prime}=\partial T$.

Proof. Take $\phi=0$ if not given. Denote $K \triangleq \operatorname{conv} \operatorname{supp} T$, and $\bar{B} \triangleq B(0, \min \{\epsilon, 1\})$. Choose a sequence $\left\{T_{i}\right\}_{i=1}^{\infty}$ of polyhedral currents with $T_{i} \stackrel{\mathbb{F}}{\rightarrow} T$, supp $T_{i} \subset K+\bar{B} / 3$, and $\mathbb{M}^{r}\left(T_{i}\right) \leqslant$ $\mathbb{M}^{r}(T)+\epsilon / 3$. Then $\mathbb{F}\left(\partial T-\partial T_{i}\right) \leqslant \mathbb{F}\left(T-T_{i}\right) \leqslant C \epsilon / 12$ for $i$ sufficiently large and $C \triangleq$ $1 /\left[r^{\prime}(0)(\operatorname{diam} K+2)\right]$. Also choose a sequence $\left\{\phi_{i}^{\prime}\right\}_{i=1}^{\infty}$ of polyhedral 0 -dimensional currents with $\phi_{i}^{\prime} \stackrel{\mathbb{F}}{\rightarrow} \partial T-\phi, \operatorname{supp} \phi_{i}^{\prime} \subset K+\bar{B} / 3$, and $\mathbb{M}\left(\phi_{i}^{\prime}\right) \leqslant \mathbb{M}(\partial T-\phi)+\epsilon$. For sufficiently large $i$, again $\mathbb{F}\left((\partial T-\phi)-\phi_{i}^{\prime}\right) \leqslant C \epsilon / 24$. Since $\phi$ is a sum of Dirac masses with $\mathbb{M}(\phi)<\infty$, we may also find a polyhedral $\phi^{\prime} \leqslant \phi$ with $\mathbb{M}(\phi) \leqslant \mathbb{M}\left(\phi^{\prime}\right)+C \epsilon / 24$.

Letting $\phi_{i} \triangleq \phi^{\prime}+\phi_{i}^{\prime}$, we find

$$
\mathbb{F}\left(\partial T-\phi_{i}\right) \leqslant \mathbb{M}\left(\phi-\phi^{\prime}\right)+\mathbb{F}\left((\partial T-\phi)-\phi_{i}^{\prime}\right) \leqslant C \epsilon / 12 .
$$

Therefore, $\mathbb{F}\left(\phi_{i}-\partial T_{i}\right) \leqslant C \epsilon / 6$. Since $\phi_{i}$ and $\partial T_{i}$ are polyhedral, this implies by [11, Lemma 4.2.23] the existence of a polyhedral current $Q_{i}$ with

$$
\mathbb{M}\left(\phi_{i}-\partial T_{i}-\partial Q_{i}\right)+\mathbb{M}\left(Q_{i}\right) \leqslant C \epsilon / 4
$$

and supp $Q_{i} \subset K+\bar{B} / 2$,

Now, consider $\psi_{i} \triangleq \phi+\phi_{i}^{\prime}-\partial T_{i}-\partial Q_{i}$. It is supported on $K+\bar{B} / 2$, and

$$
\mathbb{M}\left(\psi_{i}\right)+\mathbb{M}\left(Q_{i}\right) \leqslant \mathbb{M}\left(\phi-\phi^{\prime}\right)+\mathbb{M}\left(\phi_{i}-\partial T_{i}-\partial Q_{i}\right)+\mathbb{M}\left(Q_{i}\right) \leqslant C \epsilon / 3 .
$$

Thus $\psi_{i}^{+}\left(\mathbb{R}^{m}\right)=\psi_{i}^{-}\left(\mathbb{R}^{m}\right)=\mathbb{M}\left(\psi_{i}\right) / 2<\infty$. Since $\psi_{i}$ is also real rectifiable, i.e., a countable weighted sum of Dirac measures, we may construct a real rectifiable current $R_{i}$ with $\partial R_{i}=\psi_{i}$ and $\mathbb{M}\left(R_{i}\right) \leqslant \mathbb{M}\left(\psi_{i}\right) \operatorname{diam}(K+\bar{B})$. By convexity of $K$, we can take supp $R_{i} \subset K+\bar{B}$. Letting

$$
T_{i}^{\prime} \triangleq T_{i}+Q_{i}+R_{i}
$$

we therefore have $\operatorname{supp} T_{i}^{\prime} \subset K+\bar{B}$ and $\partial T_{i}^{\prime}=\phi+\phi_{i}^{\prime}$, whence

$$
\mathbb{M}\left(\partial T_{i}^{\prime}\right) \leqslant \mathbb{M}(\phi)+\mathbb{M}\left(\phi_{i}^{\prime}\right) \leqslant \mathbb{M}(\phi)+\mathbb{M}(\partial T-\phi)+\epsilon=\mathbb{M}(\partial T)+\epsilon .
$$


We also have $\mathbb{M}\left(T_{i}^{\prime}\right)<\infty$ and, moreover, $\mathbb{M}^{r}\left(T_{i}^{\prime}\right) \leqslant \mathbb{M}^{r}\left(T_{i}\right)+\mathbb{M}^{r}\left(Q_{i}\right)+\mathbb{M}^{r}\left(R_{i}\right)$ by subadditivity. But, since $r \leqslant r^{\prime}(0)$ id and

$$
\operatorname{Cr}^{\prime}(0)=1 /(\operatorname{diam} K+2) \leqslant 1,
$$

we get

$$
\mathbb{M}^{r}\left(Q_{i}\right) \leqslant \mathbb{M}\left(Q_{i}\right) r^{\prime}(0) \leqslant C r^{\prime}(0) \epsilon / 3 \leqslant \epsilon / 3
$$

and likewise,

$$
\mathbb{M}^{r}\left(R_{i}\right) \leqslant \mathbb{M}\left(R_{i}\right) r^{\prime}(0) \leqslant C r^{\prime}(0) \operatorname{diam}(K+\bar{B}) \epsilon / 3 \leqslant \epsilon / 3 .
$$

Thus $\mathbb{M}^{r}\left(T_{i}^{\prime}\right) \leqslant \mathbb{M}^{r}(T)+\epsilon$, and, employing 5.2 twice, we obtain

$\mathbb{F}\left(T_{i}^{\prime}-T\right) \leqslant \mathbb{F}\left(T_{i}-T\right)+\mathbb{M}\left(Q_{i}\right)+\mathbb{M}\left(R_{i}\right) \leqslant C \epsilon / 12+C \epsilon / 4+(C \epsilon / 3) \operatorname{diam}(K+\bar{B}) \leqslant \epsilon / r^{\prime}(0)$.

We have the first part of our claim.

Finally, since $\phi$ is real rectifiable, i.e., a countable sum of Dirac masses, while $\phi_{i}^{\prime}$ is a finite sum of Dirac masses, we may choose $\phi_{i}^{\prime}$ while maintaining $\phi \leqslant \phi+\phi_{i}^{\prime}$. Hence $\phi_{i} \leqslant \partial T_{i}^{\prime}=\phi+\phi_{i}^{\prime}$.

LEMMA 5.5 Suppose $r \neq 0$ and $T$ is a one-dimensional normal current. Then there exists a normal current $T^{\prime}$ with supp $T^{\prime} \subset K \triangleq \operatorname{conv} \operatorname{supp} T+B(0,1), \partial T^{\prime}=\partial T, \mathbb{M}^{r}\left(T^{\prime}\right) \leqslant \mathbb{M}^{r}(T)$, and

$$
\mathbb{M}\left(T^{\prime}\right) \leqslant \mathbb{M}^{r}(T)\left[\frac{\mathrm{id}}{r}(\mathbb{M}(\partial T) / 2)\right] .
$$

If id $\prec r$ and $\mathbb{M}^{r}(T)<\infty$, then $T^{\prime} \leqslant T$ is real rectifiable and acyclic.

Proof. If $\mathbb{M}(\partial T)=0$, we may choose $T^{\prime}=0$. Therefore, we may assume $\mathbb{M}(\partial T)>0$ and $\mathbb{M}^{r}(T)<\infty$, for otherwise there is nothing to prove.

Recall that $\mathrm{id} / r$ is non-decreasing on all of $(0, \infty)$, so $\tau=\infty$. If id $\prec r$, then Lemma 5.1 implies that $T$ is real rectifiable. We are therefore done after removing cycles from $T$, as can be done by [14, Proposition 3.12], and applying Lemma 5.3

Otherwise $0<r^{\prime}(0)<\infty$, and we may choose $\epsilon \in(0,1)$ and apply Lemma 5.4 to yield normal real rectifiable $T_{\epsilon}$ with supp $T_{\epsilon} \subset K, \mathbb{M}^{r}\left(T_{\epsilon}\right) \leqslant \mathbb{M}^{r}(T)+\epsilon, \mathbb{M}\left(\partial T_{\epsilon}\right) \leqslant \mathbb{M}(\partial T)+\epsilon$, and $\mathbb{F}\left(T_{\epsilon}-T\right) \leqslant \epsilon / r^{\prime}(0)$. We remove cycles from $T_{\epsilon}$ and denote the result by $T_{\epsilon}^{\prime}$. As $\partial T_{\epsilon}^{\prime}=\partial T_{\epsilon}$, still $\operatorname{supp} T_{\epsilon}^{\prime} \subset K, \mathbb{M}^{r}\left(T_{\epsilon}^{\prime}\right) \leqslant \mathbb{M}^{r}(T)+\epsilon, \mathbb{M}\left(\partial T_{\epsilon}^{\prime}\right) \leqslant \mathbb{M}(\partial T)+\epsilon$, and $\mathbb{F}\left(\partial T_{\epsilon}-\partial T\right) \leqslant \epsilon / r^{\prime}(0)$.

By Lemma 5.3 , we now have

$$
\mathbb{M}\left(T_{\epsilon}^{\prime}\right) \leqslant \mathbb{M}^{r}\left(T_{\epsilon}^{\prime}\right)\left[\frac{\mathrm{id}}{r}\left(\mathbb{M}\left(\partial T_{\epsilon}^{\prime}\right) / 2\right)\right] \leqslant\left(\mathbb{M}^{r}(T)+\epsilon\right)\left[\frac{\mathrm{id}}{r}((\mathbb{M}(\partial T)+\epsilon) / 2)\right] .
$$

Thus, $\mathbb{M}\left(T_{\epsilon}^{\prime}\right)$ is uniformly bounded as $\epsilon \searrow 0$, as is $\mathbb{M}\left(\partial T_{\epsilon}^{\prime}\right) \leqslant \mathbb{M}(\partial T)+\epsilon$, and $\operatorname{supp} T_{\epsilon}^{\prime} \subset K$. We may therefore take $T^{\prime}$ as the $\mathbb{F}$-limit of a subsequence of $\left\{T_{\epsilon}^{\prime}\right\}$. Note that in addition to being non-decreasing, id $/ r$ is concave, hence continuous in $(0, \infty)$. Therefore, the property $\mathbb{M}\left(T^{\prime}\right) \leqslant$ $\liminf _{\epsilon \searrow 0} \mathbb{M}\left(T_{\epsilon}^{\prime}\right)$ and (5.4) take care of 5.3.

\subsection{Instances of problem $(\mathrm{P})$}

We now consider problem $(\mathrm{P})$ in the setting

$$
J_{W}(W) \triangleq \mathbb{M}^{w}(W), \quad J_{R}(R) \triangleq \mathbb{M}^{r}(R)+\mathbb{M}^{s}(\partial R)+H\left(\mathbb{M}^{r}(R)+\mathbb{M}^{s_{H}}(\partial R)\right),
$$

where $w, r, s, r_{H}, s_{H} \in \mathcal{W}$, and $H:[0, \infty) \rightarrow[0, \infty]$ is lower-semicontinuous and nondecreasing, satisfying $H(\infty-)=\infty$. We also write $J_{R}(R)=\tilde{J}_{R}(R)+H\left(J_{H}(R)\right)$. 
THEOREM 5.1 Suppose that (i) $w \neq 0$, (ii) either id $\prec s$ and $s(\infty-)=\infty$, or id $\prec s_{H}$ and $s_{H}(\infty-)=\infty$, and (iii) either id $\prec r$, or id $\prec r_{H}$. Then there exists a solution to the problem (P) \&(J-WA) with $W$ normal and $R \in \mathcal{R}^{*}$.

Proof. If there were no solution with finite $J(W, R)$, we could take as $W$ the solution of the (1-Wasserstein distance) Monge-Kantorovich problem for $\mu$, and $R=0$. (A normal solution $W$ exists; cf., e.g., [1, 17].) So suppose $\left(W_{i}, R_{i}\right)$ is a pair of real flat chains of finite mass such that $J\left(W_{i}, R_{i}\right)<\infty$ and $\partial\left(W_{i}+R_{i}\right)=\mu$.

By the assumptions (ii) on $s$ (or $s_{H}$ ), $J_{R}\left(R_{i}\right)<\infty$ implies that $\partial R_{i}$ is rectifiable with $\mathbb{M}\left(\partial R_{i}\right)<s^{-1}\left(\mathbb{M}^{s}\left(\partial R_{i}\right)\right)<\infty$ through Lemma 5.1 Since $\mu$ is assumed to have compact support, we may assume that $R_{i}$ and $W_{i}$ are supported for all $i$ on some compact set $K$, employing a standard projection argument. Thus $W_{i}$ and $R_{i}$ are actually normal currents.

We consider the case id $\prec r$, the case id $\prec r_{H}$ being similar. By the assumptions (iii) and Lemma 5.5. we may replace $R_{i}$ with real rectifiable and acyclic $R_{i}^{\prime}$ satisfying $\partial R_{i}^{\prime}=\partial R, \mathbb{M}^{r}\left(R_{i}^{\prime}\right) \leqslant$ $\mathbb{M}^{r}\left(R_{i}\right)$ and $\mathbb{M}\left(R_{i}^{\prime}\right) \leqslant \mathbb{M}^{r}\left(R_{i}\right)\left[\frac{\mathrm{id}}{r}\left(\mathbb{M}\left(\partial R_{i}\right) / 2\right)\right] \leqslant C$ for some constant $C>0$. Since in the present case id $\prec r$, actually $R_{i}^{\prime} \leqslant R_{i}$ by the lemma, we also have $\mathbb{M}^{r} H\left(R_{i}^{\prime}\right) \leqslant \mathbb{M}^{r_{H}}\left(R_{i}\right)$. Likewise, by the assumption (i) and Lemma 5.5, we get $W_{i}^{\prime}$ with $\partial W_{i}^{\prime}=\partial W_{i}, \mathbb{M}^{w}\left(W_{i}^{\prime}\right) \leqslant \mathbb{M}^{w}\left(W_{i}\right)$, and $\mathbb{M}\left(W_{i}^{\prime}\right) \leqslant \mathbb{M}^{w}\left(W_{i}\right)\left[\frac{\mathrm{id}}{w}\left(\mathbb{M}\left(\partial W_{i}\right) / 2\right)\right] \leqslant C$. Consequently, $J\left(W_{i}^{\prime}, R_{i}^{\prime}\right) \leqslant J\left(W_{i}, R_{i}\right)$. Furthermore, by the lemma, supp $W_{i}^{\prime}$, supp $R_{i}^{\prime} \subset K+B(0,1)$.

Thus, given a minimising sequence $\left\{\left(W_{i}, R_{i}\right)\right\}_{i=1}^{\infty}$ for (P)\&(J-WA), we can find another minimising sequence $\left\{\left(W_{i}^{\prime}, R_{i}^{\prime}\right)\right\}_{i=1}^{\infty}$ with uniformly bounded mass, boundary mass, and support. Therefore, we can find a subsequence $\mathbb{F}$-convergent to some normal currents $W$ and $R$, that must solve the problem $(\mathbb{P})$ by the lower-semicontinuity of $J$ with regard to $\mathbb{F}$-convergence. Furthermore, $R$ and $\partial R$ remain rectifiable, and $R$ can be taken acyclic, by the same arguments as above.

Remark 5.1 We do not require that $H$ is concave and finite-valued, as do Paolini and Stepanov [14]; it might even be used to "cap the costs" to an interval $[0, M]$. The convergence proof here also differs from the base steps of the one in that paper, in that we do not impose rectifiability requirements on $W$ (which is where Lemma 5.4 becomes useful), and we do not force $W, R \leqslant$ $W+R$. Indeed, since the construction of stations can be relatively expensive for small amounts of traffic, one might have to "walk along the track" in the opposite direction.

By the above proof, by restricting the area of definition of $J$ to enforce compact support, $\mathrm{A}-\mathrm{N}$ can be taken to hold. Clearly $(\mathrm{A}-\mathrm{S})$ also holds since $\mathbb{M}^{w}$ is subadditive. In the remainder of this section, we intend to show (A-C) and (A-D) under various assumptions on $w, r, s, r_{H}, s_{H}$, and $H$.

Definition $5.1 r_{H}$ is compatible with $r$ if $r_{H}(t)=A r(t)+B t^{0}, A, B \geqslant 0$.

Lemma 5.6 Suppose $R \in \mathcal{R}^{*}$. Then (A-C) holds in the following cases:

(i) $r$ is strictly concave, and $r_{H}$ compatible with $r$.

(ii) $r_{H}$ is strictly concave, $r$ compatible with $r_{H}$, and $H$ strictly increasing.

(iii) $r$ is strictly concave, and $H$ concave.

(iv) $r_{H}$ and $H$ are strictly concave.

Proof. (i) Suppose $\left(C_{1}, C_{2}\right)$ is a circuit of $R$. Define $C \triangleq C_{1}-C_{2}$. Then $C=R\llcorner\sigma$ for some Borel $\sigma: \mathbb{R}^{m} \rightarrow[-1,1]$, so $R \pm C=R\llcorner(1 \pm \sigma)$. By Lemma 5.2 and the strict concavity of $r, f: t \in$ $[-1,1] \mapsto \mathbb{M}^{r}(R\llcorner(1+t \sigma))$ is strictly concave. We therefore find that $(f(-1)+f(1)) / 2<f(0)$, 
whence

$$
\min \left\{\mathbb{M}^{r}(R+C), \mathbb{M}^{r}(R-C)\right\} \leqslant \frac{1}{2}\left(\mathbb{M}^{r}(R+C)+\mathbb{M}^{r}(R-C)\right)<\mathbb{M}^{r}(R) .
$$

Since $C_{1}, C_{2} \leqslant R$, we have $\mathbb{M}^{t \mapsto t^{0}}(R \pm C) \leqslant \mathbb{M}^{t \mapsto t^{0}}(R)$. The compatibility assumption on $r_{H}$ then ensures that the minimising choice cannot increase $\mathbb{M}^{r}$. Also $\partial R=\partial(R \pm C)$, so the boundary costs are unaffected. Thus $J_{R}$ is improved by replacing $R$ by one of $R \pm C$.

(ii) This case is analogous to case (i), this time applying Lemma 5.2 with $r_{H}$. Since $H$ is strictly increasing, the decrease is transferred to $J_{R}$.

(iii) \& (iv) In these cases $f: t \in[-1,1] \mapsto J_{R}(R\llcorner(1+t \sigma))$ is strictly concave by Lemma5.2 and the concavity assumptions on $H$. A similar reasoning now shows the claim.

Lemma 5.7 Suppose $R \in \mathcal{R}^{*}$ and $w \prec r$. Then there is a $\beta>0$ such that $\mathrm{A}-\mathrm{D}$ holds for $\mathcal{B}_{\mathrm{disj}}^{\beta}$ and $\mathcal{B}_{\text {disj, } \mathrm{x}}^{\beta}$.

Proof. We first consider the case of $\mathcal{B}_{\text {disj. }}^{\beta}$. Denote the non-decrease threshold of $w / r$ by $\tau>0$. By Lemma 5.3 if $B$ is a disjoint component of $R$ with $\mathbb{M}(\partial B) / 2<\tau$ and $(w / r)(\mathbb{M}(\partial B) / 2) \leqslant 1$, then $\mathbb{M}^{w}(B) \leqslant \mathbb{M}^{r}(B)\left[\frac{w}{r}(\mathbb{M}(\partial B) / 2)\right]<\mathbb{M}^{r}(B)$. Thus, by disjointness of $B \leqslant R$ and $R-B$, $M^{w}(B)+\mathbb{M}^{r}(R-B)<\mathbb{M}^{r}(R)$. Since $\partial B \leqslant \partial R$, we have $\mathbb{M}^{s}(\partial R-\partial B) \leqslant \mathbb{M}^{s}(\partial R)$. Thus $J_{W}(B)+\tilde{J}_{R}(R-B)<\tilde{J}_{R}(R)$. We likewise get $J_{H}(R-B) \leqslant J_{H}(R)$, since in this case just $w=0$. Therefore, $J_{W}(B)+J_{R}(R-B)<J_{R}(R)$, so A-D holds.

Let now $x$ be a cut point. If $B$ is a disjoint component of $R$ in $\{x\}^{c}$, then the above estimates hold except possibly for $s$ and $s_{H}$. To show that the costs do not increase, note that subtracting $\partial B$ from $\partial R$ adds mass only at $x$ while all mass is removed from other points of img $\partial B$, since $\partial B$ and $\partial(R-B)$ split $\partial R$ in $\{x\}^{c}$. The mass removed is in total at least as much as that added at $x$, because $|B(x)| \leqslant(\partial B)^{ \pm}\left(\mathbb{R}^{m}\right)=\mathbb{M}(\partial B) / 2$. Therefore, if $\partial B=\sum_{y \in Y} m_{y} \delta_{y}$, then $\mathbb{M}^{s}(\partial R-\partial B)-$ $\mathbb{M}^{s}(\partial R) \leqslant s\left(m_{x}\right)-\sum_{y \in Y \backslash\{x\}} s\left(\left|m_{y}\right|\right) \leqslant s\left(\left|m_{x}\right|\right)-s\left(\sum_{y \in Y \backslash\{x\}}\left|m_{y}\right|\right) \leqslant 0$. An analogous estimate holds for $s_{H}$.

The next two lemmas ensure the conditions of the cases of Lemma 4.5

Lemma 5.8 Suppose $R \in \mathcal{R}^{*}, w, r \prec s$, and $r_{H} \prec s_{H}$ (or $r_{H}=s_{H}=0$ ). Then, given $\delta>0$, there is a $g:(0, \infty) \rightarrow(0, \infty)$ such that $\mathrm{A}-\mathrm{D}$ holds for $\mathcal{B}_{\text {seg }}^{\delta, g}$.

Proof. Let $x, y \in \operatorname{img} \partial R$ with $\|x-y\| \leqslant \delta$. Write $m \triangleq \partial R(x)$ and $t \triangleq \partial R(y)$. Then

$$
\begin{aligned}
J_{W}(t \llbracket x, y \rrbracket)+ & \tilde{J}_{R}(R-t \llbracket x, y \rrbracket)-\tilde{J}_{R}(R) \\
& \leqslant \mathbb{M}^{w}(t \llbracket x, y \rrbracket)+\mathbb{M}^{r}(t \llbracket x, y \rrbracket)+\mathbb{M}^{s}\left((m+t) \delta_{x}\right)-\left[\mathbb{M}^{s}\left(m \delta_{x}\right)+\mathbb{M}^{s}\left(t \delta_{y}\right)\right] \\
& =\|x-y\|(w(t)+r(t))+s(m+t)-s(m)-s(t) .
\end{aligned}
$$

This value is dominated from above by

$$
\begin{aligned}
f(t) & \triangleq \delta(w(t)+r(t))+s(m+t)-s(m)-s(t) . \\
& =s(m+t)-s(m)-s(t)\left[1-\delta \frac{w(t)+r(t)}{s(t)}\right] .
\end{aligned}
$$

Now, since $w, r \prec s$ and $m \neq 0, f(t) / t \rightarrow s^{\prime}(m)-s^{\prime}(0)=-\infty$ as $t \searrow 0$. Therefore, $f(t)<$ $f(0)=0$ for $t \in(0, \tilde{g}(m))$ and some $\tilde{g}(m)>0$. 
Similar estimates hold for $J_{H}(R-t \llbracket x, y \rrbracket)-J_{H}(R)$, yielding threshold $g_{H}(m)$. Therefore, we get the claim with $g(m)=\min \left\{\tilde{g}(m), g_{H}(m)\right\}$.

Lemma 5.9 Suppose $R \in \mathcal{R}^{*}$ and $w \prec s$. Then, given $M, \theta>0$, there is a $g:(0, \infty) \rightarrow(0, \infty)$ such that $\mathrm{A}-\mathrm{D}$ holds for $\mathcal{B}_{\mathrm{conn}}^{M, \theta}$.

Proof. With the notation of Lemmas 5.8 and 4.5 .

$$
\begin{aligned}
J_{W}(t C)+ & \tilde{J}_{R}(R-t C)-\tilde{J}_{R}(R) \\
& \leqslant \mathbb{M}^{w}(t C)+\mathbb{M}^{r}(R-t C)-\mathbb{M}^{r}(R)+\mathbb{M}^{s}\left((m+t) \delta_{x}\right)-\mathbb{M}^{s}\left(m \delta_{x}\right)-\mathbb{M}^{s}\left(t \delta_{y}\right) \\
& =\mathbb{M}^{w}(t C)+\mathbb{M}^{r}(R-t C)-\mathbb{M}^{r}(R)+s(m+t)-s(m)-s(t) .
\end{aligned}
$$

The right hand side is thus dominated as a function of $t$ by

$$
f(t) \triangleq \mathbb{M}^{r}(R-t C)-\mathbb{M}^{r}(R)+s(m+t)-s(m)-s(t)\left[1-\frac{\mathbb{M}^{w}(t C)}{s(t)}\right] .
$$

Clearly $f(0)=0$, while $f(t) / t \rightarrow \mathbb{M}^{r}(R+\cdot C)^{\prime}(0)+s^{\prime}(m)-s^{\prime}(0)=-\infty$, if we can show that $\mathbb{M}^{r}(R+\cdot C)^{\prime}(0)<\infty$ and $\lim _{t \searrow 0} \mathbb{M}^{w}(t C) / s(t)=0$.

We may assume $C_{1} \wedge C_{2}=0$, where $C \triangleq C_{1}-C_{2}$ with $\theta C_{1}, \theta C_{2} \leqslant R$. (Otherwise replace $C_{1}$ by $C_{1}-C_{1} \wedge C_{2}=C_{1} \vee C_{2}-C_{2}$, etc., recalling Lemma 3.2.) Therefore, since $R$ contains no circuit, $C$ cannot contain one either. Since $C$ is a rectifiable edge with $\partial C=\delta_{y}-\delta_{x}$, by Lemma 3.10. $C=\llbracket \gamma \rrbracket$ for some Lipschitz path $\gamma$. Furthermore, $\mathcal{H}^{1}(\operatorname{img} \gamma) \leqslant M$, so

$$
\mathbb{M}^{w}(t C)=\int_{\operatorname{img} \gamma} w(t) \mathrm{d} \mathcal{H}^{1} \leqslant w(t) \mathcal{H}^{1}(\operatorname{img} \gamma) \leqslant w(t) M .
$$

Also $R=\llbracket \Sigma \rrbracket\left\llcorner\sigma_{R}\right.$ for a rectifiable set $\Sigma$ (with implicit tangent vector field) and Borel $\sigma_{R}: \mathbb{R}^{m} \rightarrow$ $[0, \infty)$. Since img $\gamma \subset \Sigma, C=\llbracket \Sigma \rrbracket\left\llcorner\sigma_{C}\right.$ for some Borel $\sigma_{C}: \mathbb{R}^{m} \rightarrow\{-1,0,1\}$ with the estimate $\theta\left|\sigma_{C}\right| \leqslant \sigma_{R}$. Employing the fact that $r^{\prime}$ is non-negative and decreasing,

$$
\begin{aligned}
\mathbb{M}^{r}(R+\cdot C)^{\prime}(0) & =\int_{\Sigma} r\left(\sigma_{R}+\cdot \sigma_{C}\right)^{\prime}(0) \mathrm{d} \mathcal{H}^{1} \leqslant \int_{\Sigma} r^{\prime}\left(\sigma_{R}\right)\left|\sigma_{C}\right| \mathrm{d} \mathcal{H}^{1} \\
& \leqslant \int_{\operatorname{img} \gamma} r^{\prime}\left(\theta\left|\sigma_{C}\right|\right)\left|\sigma_{C}\right| \mathrm{d} \mathcal{H}^{1} \leqslant r^{\prime}(\theta) M .
\end{aligned}
$$

But $r^{\prime}(\theta)<\infty$ since $\theta>0$. Also $w(t) / s(t) \searrow 0$ as $t \searrow 0$. Therefore, the required estimates hold.

Similar estimates hold for $J_{H}$, as then essentially just $w=0$. The rest now follows by combining the estimates as in Lemma 5.8 .

Summarising, we finally get

TheOREM 5.2 Let $\mu$ be a signed measure with $\mu^{+}\left(\mathbb{R}^{m}\right)=\mu^{-}\left(\mathbb{R}^{m}\right)<\infty$. Suppose that $w, r, s, r_{H}, s_{H} \in \mathcal{W}, w \neq 0$, and that $H:[0, \infty) \rightarrow[0, \infty)$ is lower-semicontinuous and nondecreasing, satisfying $H(\infty-)=\infty$. Suppose also one of

(i) $r$ is strictly concave, and $r_{H}$ compatible with $r$,

(ii) $r_{H}$ is strictly concave, $r$ compatible with $r_{H}$, and $H$ strictly increasing,

(iii) $r$ is strictly concave, and $H$ concave,

(iv) $r_{H}$ and $H$ are strictly concave, 
as well as one of

(v) $w \prec r, s$,

(vi) $w, r \prec s, r_{H} \prec s_{H}$ (or $r_{H}=s_{H}=0$ ), and either id $\prec r$ or id $\prec r_{H}$,

along with

(vii) either $s(\infty-)=\infty$, or id $\prec s_{H}$ and $s_{H}(\infty-)=\infty$.

Then there exists an optimal solution to the problem $(\mathrm{P}) \&(\mathrm{~J}-\mathrm{WA})$, and any finitely optimal $R$ is polyhedral and contains no circuit.

Proof. The assumption $w \prec r$ implies id $\prec r$, and $w \prec s$ implies id $\prec s$. Therefore, the conditions of Theorem 5.1 hold in all cases, and any finitely optimal $R$ is in $\mathcal{R}^{*}$. The conditions of Lemmas 5.6 through 5.9 also hold in the corresponding cases of Theorem 4.1. Consequently $R$ is the Lipschitz image of a polyhedral current with no circuit. Finally, the current with the optimal weighted area between any two points is the straight line, whence $R$ is actually polyhedral.

REMARK 5.2 Actually in Lemma 5.7 instead of $w \prec r$, we only need to assume $w \preccurlyeq r$ and $(w / r)(0)<1$. Likewise in Lemma 5.8 it suffices to assume $w, r \preccurlyeq s, r_{H} \preccurlyeq s_{H}$, and id $\prec s, s_{H}$, if we allow the lemma to determine $\delta$. Similarly, $w \preccurlyeq s$ and id $\prec s$ suffices for Lemma 5.9, if $M$ is determined by it. Therefore, Theorem 5.2 can, without much effort but with some additional statement complexity, be made to hold in a slightly wider class of objective functions.

\section{A. Connectedness and BV functions}

Set $E \triangleq\left(e_{1} \wedge \cdots \wedge e_{m}\right) \wedge \mathcal{H}^{m}$, where $\left\{e_{1}, \ldots, e_{m}\right\}$ is the canonical basis of $\mathbb{R}^{m}$. Denote by $P(G)=$ $\mathbb{M}(\partial(E\llcorner G))$ the perimeter of a set $G$. Define the density

$$
\Theta^{*}(G, x) \triangleq \limsup _{r \searrow 0} \frac{\mathcal{H}^{m}(G \cap B(x, r))}{\mathcal{H}^{m}(B(x, r))},
$$

and denote the essential boundary and essential closure of $G$ by

$$
\partial^{*} G \triangleq\left\{x \in \mathbb{R}^{m} \mid \Theta^{*}(G, x)>0, \Theta^{*}\left(G^{c}, x\right)>0\right\}, \quad \bar{G} \triangleq\left\{x \in \mathbb{R}^{m} \mid \Theta^{*}(G, x)>0\right\} .
$$

See, e.g., [3, 2] for details.

Theorem A.1 Suppose $f: \mathbb{R}^{m} \rightarrow[0, \infty)$ is a function of bounded variation, and $T \triangleq E\llcorner f$. Then $A$ is a disjoint component of $T$ if and only if $A$ and $T-A$ split $T$, and $\partial A \leqslant \partial T$.

Proof. As always, let $B \triangleq T-A$. The "only if" direction holds by definition. For the "if" direction, we must show that the requirement $\partial A \wedge \partial B=0$ and, more generally, the subcurrent primitivedecomposition requirement are superfluous. Note that $\partial A \leqslant \partial T$ simply means $\mathbb{M}(\partial A)+\mathbb{M}(\partial B)=$ $\mathbb{M}(\partial T)$.

As $A$ and $B$ split $T$, we have $A=T\left\llcorner a\right.$ and $B=T\left\llcorner b\right.$ for some BV functions $a, b: \mathbb{R}^{m} \rightarrow \mathbb{R}$ with $0 \leqslant a, b \leqslant f, a+b=f$, and $a b=0 \mathcal{H}^{m}$-a.e. Furthermore, the co-area formula for BV functions (e.g., [3]) gives

$$
\mathbb{M}(\partial A)=\int_{-\infty}^{\infty} P(\{a>t\}) \mathrm{d} t,
$$

where the perimeters are finite $\mathcal{H}^{1}$-a.e. 
Denote $A_{t} \triangleq\{a>t\}$ and $B_{t} \triangleq\{b>t\}$. Since $\mathcal{H}^{m}\left(A_{t} \cap B_{t}\right)=0$, when $A_{t}$ and $B_{t}$ have finite perimeter, by [2, Proposition 1],

$$
P\left(A_{t}\right)+P\left(B_{t}\right)=P\left(A_{t} \cup B_{t}\right)+2 \mathcal{H}^{m-1}\left(\partial^{*} A_{t} \cap \partial^{*} B_{t}\right) .
$$

But $A_{t} \cup B_{t}=\{f>t\}$, so $\mathrm{A} 1$ and $\mathbb{M}(\partial A)+\mathbb{M}(\partial B)=\mathbb{M}(\partial T)$ imply $\mathcal{H}^{m-1}\left(\partial^{*} A_{t} \cap \partial^{*} B_{t}\right)=0$ for $\mathcal{H}^{1}$-almost all $t$.

Let now $S \leqslant T$ be normal, i.e., $S=E\left\llcorner s\right.$ for a BV function $s: \mathbb{R}^{m} \rightarrow \mathbb{R}$ (supported on a compact set). That $A \wedge S$ and $B \wedge S$ split $S$ is automatic as before, as is the boundaries summing to $\partial S$, assuming normality of the former. We thus have to show that, moreover, $\mathbb{M}(\partial(A \wedge S))+$ $\mathbb{M}(\partial(B \wedge S))=\mathbb{M}(\partial S)$ and $\partial(A \wedge S) \wedge \partial(B \wedge S)=0$

We have $A \wedge S=E\llcorner(a \wedge s)$. Thus, for the first requirement it suffices by the co-area formula to show that $P(\{a \wedge s>t\})+P(\{b \wedge s>t\})=P(\{s>t\})$ for $\mathcal{H}^{1}$-almost all $t$. Since $s \leqslant a$ a.e. when $a>0$, we have $\{a \wedge s>t\}=\{s>t\} \cap\{a>u\}$ up to an $\mathcal{H}^{m}$-negligible set for any $0<u \leqslant t$. We take $u$ such that $A_{u}$ and $B_{u}$ have finite perimeter and satisfy $\mathcal{H}^{m-1}\left(\partial^{*} A_{u} \cap \partial^{*} B_{u}\right)=0$. This and the fact $\mathcal{H}^{m}\left(A_{u} \cap B_{u}\right)=0$ force $\mathcal{H}^{m-1}\left(\partial^{*}\left(A_{u} \cap S_{t}\right) \cap \partial^{*}\left(B_{u} \cap S_{t}\right)\right)=0$ by the definition of the essential boundary. Thus, by another application of [2, Proposition 1], we get the required

$$
P\left(A_{u} \cap S_{t}\right)+P\left(B_{u} \cap S_{t}\right)=P\left(\left(A_{u} \cap S_{t}\right) \cup\left(B_{u} \cap S_{t}\right)\right)=P\left(S_{t}\right) .
$$

As for the intersection, it suffices to show that the total variation measures $\mu_{\partial(S \wedge A)}$ and $\mu_{\partial(S \wedge B)}$ are mutually singular. By the co-area formula, these can be written in the forms

$$
\mu_{\partial A}(\Omega)=\int_{-\infty}^{\infty} P\left(A_{t} \mid \Omega\right) \mathrm{d} t=\int_{0}^{\infty} \mathcal{H}^{m-1}\left(\partial^{*} A_{t} \cap \Omega\right) \mathrm{d} t
$$

noting that for $t<0, A_{t}=\mathbb{R}^{m}\left(\bmod \mathcal{H}^{m}\right)$, whence $\mathcal{H}^{m-1}\left(\partial^{*} A_{t}\right)=0$.

Let $\tilde{A} \triangleq \bigcup_{u>0} \bar{A}_{u}$. Note that $\mu_{\partial A}(\tilde{A})=\int \mathcal{H}^{m-1}\left(\partial^{*} A_{t}\right) \mathrm{d} t=\mathbb{M}(\partial A)$ by the definitions of the essential boundary and closure. Thus $\mu_{\partial A}$ is concentrated on $\tilde{A}$, and it suffices to show that $\mathcal{H}^{m-1}\left(\partial^{*} A_{t} \cap \tilde{B}\right)=0$ (for almost all $t$ ). This follows if $\mathcal{H}^{m-1}\left(\partial^{*} A_{t} \cap \bar{B}_{u}\right)=0$ for the almost all $0<u \leqslant t$ satisfying $\mathcal{H}^{m-1}\left(\partial^{*} A_{u} \cap \partial^{*} B_{u}\right)=0$. Consider first $x \in \partial^{*} A_{t} \cap \partial^{*} B_{u}$. This implies $0<$ $\Theta^{*}\left(B_{u}, x\right) \leqslant \Theta^{*}\left(A_{u}^{c}, x\right)$, and $0<\Theta^{*}\left(A_{t}, x\right) \leqslant \Theta^{*}\left(A_{u}, x\right)$. Thus $x \in \partial^{*} A_{u}$. But $\mathcal{H}^{m-1}\left(\partial^{*} A_{u} \cap\right.$ $\left.\partial^{*} B_{u}\right)=0$. Next, suppose $x \in\left(\overline{B_{u}} \backslash \partial^{*} B_{u}\right) \cap \partial^{*} A_{t}$. Then $\Theta^{*}\left(B_{u}, x\right)>0$ but $0=\Theta^{*}\left(B_{u}^{c}, x\right) \geqslant$ $\Theta^{*}\left(A_{u}, x\right) \geqslant \Theta^{*}\left(A_{t}, x\right)$. Thus $x \notin \partial^{*} A_{t}$. The claim follows.

Corollary A.1 When $T^{\prime}$ is a set of finite perimeter, $E\left\llcorner\chi_{T^{\prime}}\right.$ being connected per Definition 3.2 is equivalent to the non-existence of sets $A^{\prime}, B^{\prime}$ with $\mathcal{H}^{m}\left(T^{\prime} \triangle\left(A^{\prime} \cup B^{\prime}\right)\right)=0, \mathcal{H}^{m}\left(A^{\prime} \cap B^{\prime}\right)=0$, $\mathcal{H}^{m}\left(A^{\prime}\right), \mathcal{H}^{m}\left(B^{\prime}\right)>0$ and $P\left(A^{\prime}\right)+P\left(B^{\prime}\right)=P\left(T^{\prime}\right)$.

Thus, in this case, connectedness is equivalent to indecomposability in the sense of [3, 2] up to $\mathcal{H}^{m}$-negligible sets. (In these references $\left\{A^{\prime}, B^{\prime}\right\}$ is a partition of $T^{\prime}$.)

\section{REFERENCES}

1. Ambrosio, L. Lecture notes on optimal transport problems. In: Mathematical Aspects of Evolving Interfaces, Lecture Notes in Math. 1812, Springer (2003), 1-52. Zbl 1047.35001 MR 2011032

2. Ambrosio, L., Caselles, V., Masnou, S., \& Morel, J. Connected components of sets of finite perimeter and applications to image processing. J. Eur. Math. Soc. 3 (2001), 39-92. Zbl 0981.49024 MR 1812124 
3. Ambrosio, L., Fusco, N., \& Pallara, D. Functions of Bounded Variation and Free Discontinuity Problems. Oxford Univ. Press (2000). Zbl 0957.49001 MR 1857292

4. Aubin, J.-P., \& Frankowska, H. Set-Valued Analysis. Birkhäuser (1990). Zbl 0713.49021 MR 1048347

5. Bernot, M., Caselles, V., \& Morel, J.-M. The structure of branched transportation networks. Calc. Var. Partial Differential Equations 32 (2008), 279-317. Zbl 1146.49035 MR 2393069

6. Brancolini, A., \& Buttazzo, G. Optimal networks for mass transportation problems. ESAIM Control, Optim. Calc. Var. 11 (2005), 88-101. Zbl 1103.49002 MR 2110615

7. Buttazzo, G., Pratelli, A., Solimini, S., \& Stepanov, E. Optimal Urban Networks via Mass Transportation. Lecture Notes in Math. 1961, Springer (2009). Zbl pre05349205 MR 2469110

8. Buttazzo, G., \& StePanov, E. Minimization problems for average distance functionals. In: Calculus of Variations: Topics from the Mathematical Heritage of Ennio De Giorgi, D. Pallara (ed.), Quad. Mat. 14, Seconda Università di Napoli, 47-83 (2004). Zbl 1089.49040 MR 2118415

9. Castaing, C., \& Valadier, M. Convex Analysis and Measurable Multifunctions. Springer (1977). Zbl 0346.46038 MR 0467310

10. De PAuw, T., \& HARDT, R. Size minimization and approximating problems. Calc. Var. Partial Differential Equations 17 (2003), 405-442. Zbl 1022.49026 MR 1993962

11. FedERER, H. Geometric Measure Theory. Springer (1969). Zbl 0176.00801 MR 0257325

12. Himmelberg, C. J. Measurable relations. Fund. Math. 87 (1975), 53-72. Zbl 0296.28003 MR 0367142

13. Morgan, F. Geometric Measure Theory: A Beginner's Guide. Academic Press, 1988. Zbl 0671.49043 MR 0933756

14. Paolini, E., \& Stepanov, E. Optimal transportation networks as flat chains. Interfaces Free Bound. 8 (2006), 393-436. Zbl pre05081442 MR 2283920

15. White, B. The deformation theorem for flat chains. Acta Math. 183 (1999), 255-271. Zbl 0980.49035 MR 1738045

16. White, B. Rectifiability of flat chains. Ann. of Math. 150 (1999), 165-184. Zbl 0965.49024 MR 1715323

17. XiA, Q. Optimal paths related to transport problems. Comm. Contemp. Math. 5 (2003), 251-280. Zbl 1032.90003 MR 1966259

18. XIA, Q. Boundary regularity of optimal transport paths. Preprint (2004).

19. XIA, Q. Interior regularity of optimal transport paths. Calc. Var. Partial Differential Equations 20 (2004), 283-299. Zbl 1080.90008 MR 2062945 